\title{
Kernos
}

Revue internationale et pluridisciplinaire de religion grecque antique

$30 \mid 2017$

Varia

\section{De Thalès à Anaxagore : les Ioniens à l'école des dieux}

\section{Pierre Vesperini}

\section{(2) OpenEdition \\ Journals}

\section{Electronic version}

URL: http://journals.openedition.org/kernos/2486

DOI: 10.4000/kernos.2486

ISSN: 2034-7871

\section{Publisher}

Centre international d'étude de la religion grecque antique

\section{Printed version}

Date of publication: 1 October 2017

Number of pages: 37-65

ISSN: 0776-3824

\section{Electronic reference}

Pierre Vesperini, « De Thalès à Anaxagore : les Ioniens à l'école des dieux », Kernos [Online], 30 | 2017, Online since 01 October 2019, connection on 17 September 2020. URL : http:// journals.openedition.org/kernos/2486 ; DOI : https://doi.org/10.4000/kernos.2486

This text was automatically generated on 17 September 2020 .

Kernos 


\title{
De Thalès à Anaxagore : les Ioniens à l'école des dieux
}

\author{
Pierre Vesperini
}

\section{À Michel Vinaver}

Cet article est issu d'une conférence donnée dans le cadre du séminaire de François de Polignac, "Religions et institutions dans le monde grec ", École Pratique des Hautes Études (23 avril 2013). Entre-temps est parue aux États-Unis et en France la splendide et impressionnante édition des Présocratiques procurée par André LAKS et Glenn W. MOST, qui met à la disposition du public le plus large, entre autres découvertes postérieures au recueil de Diels, le Papyrus de Strasbourg et le Papyrus de Derveni, ainsi que de nouveaux témoignages en arabe, en hébreu, en syriaque et en arménien. Mais les éditeurs ont choisi de ne pas recueillir tous les témoignages sur les Présocratiques, tels qu'on les trouve dans le recueil de Diels: « Les témoignages sur la doctrine [...] et les réactions critiques, les interprétations et les utilisations [...] ont en revanche [sc. par opposition aux " fragments de l'œuvre originale »] été sélectionnés en fonction de leur qualité intrinsèque et de leur représentativité» (p. 9, je souligne). Je ne m'explique pas cette décision. Les jugements sur la "qualité intrinsèque » et la " représentativité » sont forcément subjectifs. Les éditeurs, je crois, en conviendront eux-mêmes. Un cinquième des témoignages utilisés dans cet article ne se trouvent par exemple pas dans leur recueil. Il serait donc bon, dans les prochaines éditions, de recueillir l'ensemble des témoignages. En attendant, si l'on veut étudier un présocratique en prenant en compte l'ensemble des témoignages, il faudra utiliser à la fois l'édition Diels-Kranz et l'édition Laks-Most.

1 Le « tournant culturel » qui depuis un demi-siècle a bouleversé et renouvelé les études d'histoire des sciences, de la philosophie, des savoirs, aux époques médiévale, moderne et contemporaine, ne semble pas avoir encore atteint l'histoire des sciences, de la philosophie, des savoirs, dans l'Antiquité. Alors que le "grand récit » d'une modernité se construisant à partir d'un combat héroïque de «la» rationalité (scientifique, philosophique, savante) contre «la» religion, n'a plus cours, l'histoire de la philosophie, des sciences et des savoirs en Grèce continue d'affirmer que «la» rationalité grecque s'est construite en dehors de « la » religion, voire dans une critique 
de « la » religion. Bien plus, après quelques timides remises en cause, ce « récit » a été ces dernières années réaffirmé avec plus de vigueur que jamais.

2 À partir d'une étude de cas, cet article voudrait contribuer à amorcer une discussion sur deux questions. Il s'agirait d'abord de comprendre pourquoi l'Antiquité reste encore aujourd'hui à l'écart du «tournant culturel». Ensuite, il s'agirait de montrer comment, en contextualisant les pratiques grecques qualifiées aujourd'hui de "scientifiques », « philosophiques », « savantes », on peut en rendre compte autrement qu'en transposant dans l'Antiquité le récit héroïque de la Modernité.

3 L'exemple choisi est un chapitre central du grand récit de la naissance de « la » Raison en Grèce, puisqu'il s'agit de ce qu'on appelle «l'école ionienne », c'est-à-dire des "philosophes" qui, de Thalès à Anaxagore, auraient fondé la philosophie en émancipant leur savoir de « la » religion.

\section{Les loniens dans le récit de la Raison grecque}

4 Essayons pour commencer de formuler la base du "grand récit » qui lie les Ioniens à la naissance de «la» philosophie. Il repose sur un postulat: «la philosophie» a été inventée en Grèce; et sur une définition: "la philosophie» se définit comme une activité consistant à penser en recourant exclusivement à la Raison (par opposition à la foi, à l'autorité, à la tradition, aux pouvoirs, etc.). Par conséquent, on peut dater la naissance de la philosophie du moment où des Grecs, en Ionie, ont rejeté la "pensée religieuse »: les termes dans lesquels cette "pensée religieuse» se formule (dieux, héros, «mythes » et tout ce que nous considérons comme relevant de l' "imaginaire " ou de l' «irrationnel»), mais aussi l'autorité des discours et des actes formulant explicitement ou non cette pensée (révélations oraculaires, « mythes ", rituels, normes traditionnelles). À la place, les Ioniens auraient proposé des explications ne se formulant qu'en termes " positifs » ou " naturalistes », ne faisant reposer leur autorité que sur le caractère rationnel de leur démonstration, cette démonstration étant exposée publiquement, s'offrant ainsi à une discussion publique. Si Thalès, premier des Ioniens, est le premier des philosophes, c'est que, le premier, il attribua à l'univers une cause matérielle, l'eau'.

5 Ainsi serait née « la philosophie ", mais aussi « la science ", non seulement en Grèce, mais dans le monde en général : par un passage « du mythe à la Raison », du muthos au logos, selon la formule du livre de Wilhelm Nestle, publié à Stuttgart en 1940 (nous y reviendrons).

6 Selon ce récit, c'est la Nature, la phusis, qui fit l'objet des explications rationnelles des Ioniens, et des " premiers philosophes » en général. Dans un deuxième temps, Socrate, en «faisant descendre la philosophie du ciel sur la terre", prendra l'Homme pour objet. L'importance de cette coupure socratique, véritable deuxième naissance de la philosophie, fait qu'on attribue aux premiers philosophes le nom de " Présocratiques » 2.

7 Ce récit remonte aux leçons sur l'histoire de la philosophie de Hegel ${ }^{3}$. C'est lui que nous retrouvons non seulement dans les manuels et les ouvrages de vulgarisation, mais aussi chez la plupart des historiens de la philosophie antique et des antiquisants, à commencer par Eduard Zeller, dont la monumentale et fondatrice histoire de la philosophie antique s'inscrit explicitement dans l'héritage de Hegel ${ }^{4}$. Pour employer le 
langage de l'archéologie foucaldienne, la philosophie de l'Histoire hégélienne constitue le «sol » à partir duquel les antiquisants (parmi les plus grands, citons Diels, Burnet, Guthrie, Snell, Gernet, Bollack, Vernant, Laks) rendirent compte de la naissance de la Raison en Grèce, ainsi que la plupart des grands penseurs de la culture, de Nietzsche, qui rapproche Thalès de Lavoisier et de Laplace ${ }^{5}$, à Pierre Bourdieu ${ }^{6}$,

en passant par Ernst Cassirer ${ }^{7}$, Max Weber ${ }^{8}$, Hans Blumenberg', et Cornelius Castoriadis $^{10}$ : tous ont admis l'idée que notre conception de la philosophie provient de la philosophie grecque et que celle-ci était née d'un passage du mythe à la Raison ${ }^{11}$.

Le caractère le plus frappant de ce récit, c'est sa persistance : Jean-Pierre Vernant, qui commence son célèbre article de 1957 par un développement critique sur le «miracle grec », finit non pas par rejeter l'idée d'un miracle grec, mais par en expliquer les conditions de possibilité, c'est-à-dire la cité, avec son espace public ${ }^{12}$. Autrement dit, il n'élimine pas le miracle grec, il le déplace: le miracle n'est plus l'apparition de la pensée rationnelle, mais l'apparition de la cité, qui va permettre l'apparition de la pensée rationnelle. Vernant critique même les travaux de Cornford ${ }^{13}$, qui pourtant ne remettait nullement en cause le dogme du rationalisme grec $^{14}$, pour n'avoir pas suffisamment distingué les premiers penseurs grecs de la pensée religieuse orientale: «rétablir le fil de la continuité historique» ne doit pas faire perdre de vue «le véritablement nouveau : ce par quoi la philosophie cesse d'être le mythe pour devenir philosophie [...], la mutation mentale » qui s'est opérée en Ionie ${ }^{15}$. On pouvait donc se demander, avec Maurice Caveing, si le miracle grec, évacué par la porte, ne rentrait pas par la fenêtre ${ }^{16}$.

9 Plus récemment furent publiés les actes d'un colloque réunissant dix-sept savants de renom, organisé en 1996 autour du titre From Myth to Reason ${ }^{17}$. À l'exception de Claude Calame $^{18}$, les antiquisants répondaient à la question posée par un oui, assorti de quelques ajustements : en un mot, les choses n'étaient pas aussi simples que les avait pensées Wilhelm Nestle en 1940 : il y avait du logos dans le muthos, et du muthos dans le $\operatorname{logos}^{19}$, mais quoi qu'il en soit, pour le dire avec Walter Burkert, «there was a unique development that brought about Greek philosophy and science, something which arose nowhere else and at no other time in just this form, but which has been kept alive until the present day by an uninterrupted tradition of books and of 'schools' reading and discussing these very books ${ }^{20}$ ».

Même le fait que Nestle ait été membre du parti nazi ne semble pas avoir conduit à remettre en question cette idée que "la philosophie » est née d'un passage « du mythe à la Raison ». Un seul des intervenants, Jan Bremmer, attira l'attention sur ce point : Nestle, alors qu'il était à la retraite et n'avait donc aucune raison de servir le régime par opportunisme, avait été le contributeur régulier d'une revue intitulée Aus Unterricht und Forschung: Wissenschaftliche Zeitschrift auf national-sozialistischer Grundlage [«Enseignement et recherche. Revue scientifique nationale-socialiste»]: il y publia notamment un article sur les juifs dans le monde gréco-romain (« Die Juden in der griechisch-römischen Welt ", 1935, p. 165 sq.) et un autre consacré à la " conception du Führer dans la doctrine politique platonicienne et aristotélicienne» («Der Führergedanke in der platonischen und aristotelischen Staatslehre », 1937, p. 73 sq.). Dans l'introduction même de son livre consacré à la naissance de la philosophie grecque, Nestle écrivait qu'il n'avait été donné qu'aux Aryens (les Grecs étant des Aryens) de parcourir ce chemin du mythe à la Raison, et que si Luther avait osé traiter la Raison d' "aveugle putain", c'était seulement parce qu'il était influencé par la conception juive selon laquelle l'aspiration des hommes à la connaissance était impie ${ }^{21}$. 
11 Ces informations, apportées au colloque par Jan Bremmer, ne suscitèrent aucun commentaire, ni dans l'introduction de Buxton au volume des actes, ni dans la contribution de Glenn Most, consacrée pourtant à la critique du logos au nom du muthos dans la philosophie allemande de Nietzsche à Adorno. Most alla même jusqu'à dire que «Nestle n'était pas du tout nazi » et prétendait par son livre «contribuer à l'accroissement de la rationalité dans le monde troublé qui l'environnait ${ }^{22}$. André Laks, tout en citant "en passant $»^{23}$ la phrase sur les Aryens dans son livre sur la "philosophie présocratique », écrit que la « compromission personnelle » de Wilhelm Nestle avec le nazisme ne change rien à la pertinence de son idée ${ }^{24}$. Et en effet, l'idée d'un passage en Grèce « du mythe à la Raison » n'est pas en elle-même une idée nazie. Mais le fait qu'elle ait été défendue en cohérence avec la nouvelle normativité promue par le régime nazi aurait dû conduire à s'interroger, non pas sur le caractère nazi de cette idée, mais sur son origine: la philosophie de l'Histoire allemande. Car la « höchstbegabte Rasse » de Nestle est en fait une transposition, dans l'idéologie nazie, du Volksgeist de Zeller et de Hegel, comme l'a montré récemment dans un magistral ouvrage un jeune philosophe allemand, interrogeant de façon critique, dans la lignée de Nietzsche, de Feyerabend et de l'école de Francfort, l'histoire de sa propre discipline ${ }^{25}$.

Rien, même l'engagement nazi d'un de ses défenseurs les plus ardents, ne semble donc pouvoir justifier que l'on aborde de façon critique le récit du passage du mythe à la Raison.

Dès lors, après la timide remise en cause représentée par le colloque de Bristol, c'est sans surprise que l'on voit en 2011 l'éminent helléniste Robert L. Fowler publier dans le Journal of Hellenic Studies un article expliquant que, mutatis mutandis, muthos correspond " in important ways" à la notion moderne de "mythe" et logos, qui correspond symétriquement à la notion moderne de Raison, "stands at the beginning of an unbroken tradition of Western rationalism ${ }^{26} »$.

On aurait pu s'attendre à ce que Marcel Detienne, après avoir critiqué Jean-Pierre Vernant pour n'être pas sorti du mythe du «miracle grec » et du passage du mythe à la raison $^{27}$, s'attaque à cette question. Mais il ne l'a jusqu'à présent pas fait: il a déconstruit la catégorie de "mythe ", mais il n'a pas déconstruit la catégorie de «Raison». Il a montré par les entreprises comparatives qu'il a dirigées que la " démocratie » n'était pas le monopole des Grecs, mais il n'a pas dirigé cette entreprise comparatiste sur le monopole de «la Raison» par «les Grecs». Sa postface à la réédition des Maîtres de vérité ne revient pas sur le schéma selon lequel la philosophie serait le produit d'un processus de «laïcisation $»^{28}$. Et au seuil de son livre sur Apollon, alors même qu'il annonce qu'il va démolir le mythe winckelmannien d'un Apollon aimable et doux, il prévient ${ }^{29}$ : «Qu'on se rassure. Il n'est pas question de faire oublier l'Apollon enjoignant à Socrate de pratiquer un genre de vie " philosophique » fondé sur la connaissance de soi et l'art de se questionner tout en questionnant autrui. » La furia antropologica épargne donc l'histoire de la philosophie comme Attila épargnait Rome, et l'ironie de l'expression "qu'on se rassure" masque mal le malaise qui entoure l'arbitraire apparent de cette décision ${ }^{30}$.

Très rares sont ceux qui ont voulu réellement remettre en cause cette dogmatique. Peter Kingsley s'y est efforcé, mais en travaillant sur des personnages tels que Pythagore ou Empédocle, que les spécialistes récents situent à la marge de l'histoire de la philosophie antique, quand ils ne les en excluent pas ${ }^{31}$. Dès lors, P. Kingsley pouvait à loisir mettre en lumière ce qu'il appelait leur «mysticisme» (par opposition au 
rationalisme prétendu des premiers philosophes), ses collègues lui concédaient bien volontiers ces originaux. Un autre savant, Giorgio Colli, philologue gigantesque et solitaire de l'université de Florence, connu en France pour son édition de Nietzsche, entreprit seul de former un nouveau corpus, intitulé La Sagesse grecque, destiné à remplacer le recueil des Présocratiques qui fait autorité, celui de Hermann Diels, révisé ensuite par Walter Kranz. Diels pensait comme Hegel que les incunables de la philosophie avaient été écrits à Milet au $\mathrm{vI}^{\mathrm{e}}$ siècle avant notre ère, comme il le proclamait encore solennellement quelques semaines avant sa mort, au milieu du désastre de l'Europe ${ }^{32}$. Nul mieux que lui ne pouvait incarner l'idéal du scientifique et de l'intellectuel moderne : en 1914, il avait refusé de signer l'Appel des 93, dans lequel les gloires de la science (y compris Wilamowitz) et de l'intelligentsia allemandes légitimaient l'invasion de la Belgique et dénonçaient la France et l'Angleterre pour avoir « lâché » contre l'Allemagne "des Nègres et des Mongols " ${ }^{33}$. Après le désastre de la guerre, de façon significative, il consumait ses dernières forces dans l'édition, la traduction et le commentaire du de rerum natura, dont Albert Einstein (qui lui aussi avait refusé de signer l'Appel des 93) devait écrire la préface et que Franz Cumont devait (en secret) financer ${ }^{34}$.

C'est donc "contre» le monument de Diels que Colli édifiait sa Sagesse grecque. Les Présocratiques de Diels commençaient par les "Sept Sages ", avant de passer à Thalès, le véritable fondateur de la philosophie. Colli commençait, lui, par un magistral premier volume dont les chapitres étaient intitulés "Dionysos ", "Apollon ", "Éleusis", «Orphée ", "Musée », "Hyperboréens ». C'est seulement dans le deuxième volume qu'apparaissait Thalès. Il avait donc réuni dans le premier volume des témoignages et des fragments d'une « sagesse grecque " placée sous le signe de deux dieux (Dionysos et Apollon) et représentée par des personnages fabuleux en lien avec ces dieux (Orphée et Musée, et ceux qu'il appelait les "Hyperboréens ", c'est-à-dire les personnages de sage venus de la Mer Noire, et qu'on appelle aussi parfois, à la suite d'E.R. Dodds ${ }^{35}$, les "chamanes grecs»). Colli montrait en effet non pas seulement que la "sagesse grecque » avait une origine religieuse, c'est-à-dire (car lui aussi continuait à employer ce vocabulaire) "irrationnelle ", "mystique», «mythique», origine religieuse dont elle allait progressivement se détacher pour devenir "philosophie", mais que cette origine constituait aussi une dimension qui allait accompagner la "sagesse grecque " pendant toute l'histoire de la philosophie grecque. Ce premier volume, avec ses commentaires magistraux et son apparat critique extrêmement riche, était une sorte de bombe placée sous le fauteuil de l'historiographie post-hégélienne ; elle l'ignora ${ }^{36}$.

Pourtant, Colli n'allait pas jusqu'à nier l'essence rationnelle de la philosophie. Il restait en ce sens fidèle à l'historiographie traditionnelle de la philosophie antique et de la philosophie en général. Seulement, il estimait que cette activité s'exerçait dans une sorte de corps à corps permanent avec l'irrationnel, le mystique, le divin. Son attention à la sagesse «dionysiaque » et à la sagesse « apollinienne » était de bout en bout une attention de philosophe, et non d'anthropologue ou d'historien. Il ne tenait pas compte de la variété des visages et des modalités d'action de Dionysos ou d'Apollon, tels qu'ils se manifestent dans les histoires, les représentations et les rituels, variété telle qu'il est impossible de réduire ces dieux (et cela vaut pour tous les dieux du polythéisme grec) à une définition essentialiste. Au contraire, comme Nietzsche, il assimilait Dionysos à une certaine idée de la sagesse, Apollon à une autre : en un mot, il les conceptualisait. 
18 J'ai voulu, dans cette première partie, faire simplement constater cette unanimité profonde de tant de grands savants, quelles que soient leurs oppositions et parfois leurs affrontements, autour de l'idée d'une philosophie grecque malgré tout, envers et contre tout, « rationnelle », " areligieuse » et « amythique », ainsi que l'échec des tentatives de penser autrement les origines de ce que nous appelons "philosophie». Comment l'expliquer, à l'heure où toutes les autres périodes de l'histoire des sciences, des savoirs, de la philosophie, font l'objet d'un tel renouvellement historiographique?

Je passe maintenant à l'exemple choisi pour amorcer un débat sur ce récit de la naissance de la Raison en Grèce : les Ioniens. Je commencerai par dire un mot de la méthode suivie: la démarche de l'historien, quand elle prend pour objet une société aussi différente de la nôtre que celle de la Grèce antique, implique aussi une démarche d'anthropologue. Or adopter une démarche d'historien anthropologue, c'est être nominaliste. C'est donc commencer par refuser le postulat selon lequel quelque chose comme « la philosophie » aurait existé à travers les siècles, depuis l'Ionie du $\mathrm{VI}^{\mathrm{e}}$ siècle avant notre ère. C'est aussi refuser cette même permanence aux catégories à partir desquelles nous pensons la philosophie aujourd'hui - «rationnel » vs. " irrationnel », «Raison"vs. " mythe », " philosophie » vs. "religion» - et partir à la rencontre de personnages du passé, qui ne nous sont rien, qui se présentent à nous comme des étrangers, en essayant de comprendre ce qu'ils faisaient à partir de leur propres catégories, de leurs propres mots. Comme l'observait très justement Richard Buxton dans son introduction au volume des actes du colloque de Bristol, renoncer au schéma du mythe et de la Raison, c'est se retrouver "sans mots ${ }^{37}:$ "We might seem to have reached a point where not only does 'the Greek achievement' have about it more of the mirage than the miracle, but where we are actually left without a vocabulary for describing the events which were once thought to constitute that achievement." Cet aveu d'impuissance, remarquable par son honnêteté intellectuelle, n'explique pas simplement pourquoi ce vieux schéma a la vie dure. Il invite aussi à tenter une nouvelle méthode d'approche. Elle doit consister précisément à accepter de se retrouver « without a vocabulary " et à aller écouter les mots des autres.

\section{Les loniens à l'école des dieux}

Nous l'avons dit: choisir pour objet d'étude les Ioniens, et non Pythagore ou Empédocle, comme Peter Kingsley, c'est porter la critique au cœur même de la vulgate, en étudiant ses deux figures les plus cruciales $^{38}$ : Thalès de Milet, le «premier philosophe ", fondateur de ce qu'on appelle "l'école ionienne» et Anaxagore de Clazomènes, le dernier représentant de cette école, qui, en s'installant à Athènes à l'époque de Périclès, fait office de chaînon reliant l'école d'Ionie à Athènes ${ }^{39}$. Et nous montrerons que l'un et l'autre, donc le fondateur du mouvement ionien et celui qui est son point d'aboutissement, loin d'avoir " laissé les dieux à la porte ", selon une formule qu'on rencontre partout ${ }^{40}$, ne pouvaient pas se concevoir, ni eux ni leur savoir ni leur "pensée », sans les dieux.

Reconnaissons-le d'emblée : les témoignages que nous possédons sur Thalès comme sur Anaxagore sont fragmentaires et souvent obscurs (en particulier pour Thalès). J'espère néanmoins qu'en les étudiant pour eux-mêmes, c'est-à-dire sans chercher à y voir les ancêtres de nos propres pratiques théoriques (qu'elles se donnent comme philosophiques ou scientifiques), et en les prenant comme des témoignages référant 
non pas à la réalité objective de Thalès et d'Anaxagore, mais aux représentations qui leur étaient rattachées, je pourrai démontrer que, bien loin d'avoir laissé les dieux à la

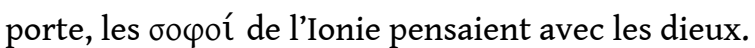

\section{Thalès}

\section{Thalès à l'école d'Apollon}

Thalès, comme Anaximandre ${ }^{41}$, autre "philosophe ionien ", appartenait, disait-on, à une famille noble, les Thelidai, qui disait descendre de Cadmos, le fondateur mythique de Thèbes $^{42}$. Mais Hérodote dit qu'il était d'extraction phénicienne ${ }^{43}$, et il n'est pas certain qu'il ait voulu par là faire allusion au fait que Cadmos, fils d'un roi de Tyr, était " phénicien $~_{44}$. Rien de certain, donc, sur ses origines. Il n'est pas davantage possible de le situer précisément dans le temps : on peut juste dire qu'il vivait à Milet à l'époque de Crésus, donc dans ce qui était encore, avant sa destruction par les Perses en 494 av. J.C., la "parure de l'Ionie $»^{45}$ : une cité extrêmement prospère ${ }^{46}$, métropole de 90 colonies $^{47}$, possédant à Didymes un oracle international, sur lequel nous reviendrons. Ce qu'on peut dire, en revanche, c'est qu'il fut reconnu dès son vivant comme un sage, un бoৎóc. En témoignent un fragment d'Alcée ${ }^{48}$ et La Liste des archontes de Démétrius de

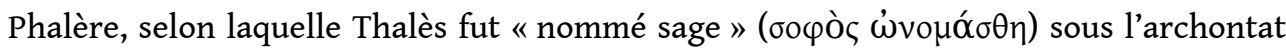
de Damasios (586/585 av. J.-C.) ${ }^{49}$.

Qu'est-ce qu'un бoৎóc, à l'époque de Thalès ? C'est, avant toutes choses, un homme qui voit l'invisible: le passé, le présent et l'avenir ${ }^{50}$; la solution d'une énigme impénétrable ${ }^{51}$; mais aussi ce qui se cache à l'intérieur des choses, leur nature $(\varphi v ́ \sigma \varsigma)^{52}$. Il est seul à voir clair dans un monde où les autres hommes passent leur temps à trébucher. Cette vision se produit de trois façons différentes: par possession, par intuition directe sans état de possession, mais aussi par conjecture (au moyen de signes, d'indices qu'on reconnaît). Ces trois façons de "voir» ne s'excluent pas, comme le montre la figure du sage Théoclymène dans l'odyssée $e^{53}$.

Or cette capacité de vision n'est pas le résultat des études ou des recherches du бoధóৎ:

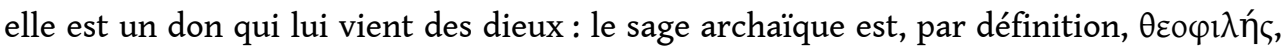
aimé des dieux ${ }^{54}$, et il n'a donc aucune raison de les «mettre à la porte ».

Thalès ne fait pas exception à cette règle : il est lui aussi en relation avec les dieux, et en particulier avec Apollon, qui "possède Milet", comme le dit l'hymne homérique ${ }^{55}$. Plusieurs témoignages le montrent.

Il y a d'abord l'histoire du trépied que des pêcheurs de Milet trouvent dans la mer ${ }^{56}$. Les Milésiens interrogent l'oracle d'Apollon, à Delphes : à qui le trépied doit-il revenir ? Réponse : "À celui qui est le premier par la бopía. » Ils donnent le trépied à Thalès. Thalès le donne à un autre sage, et le trépied commence ainsi un circuit de sage en sage qui l'amène à Solon, lequel décide que le premier par la sagesse, c'est le dieu lui-même, et envoie par conséquent le trépied à Delphes. Dans une autre version de cette histoire, racontée par Callimaque (qui prend pour source un auteur milésien nommé Léandrios ${ }^{57}$ ), l'objet n'est pas un trépied, mais une coupe en or, qu'un Arcadien nommé Bathyclès lègue " au plus utile parmi les sages ". Là aussi, on la donne à Thalès, qui la donne à un autre sage, et le circuit recommence, mais cette fois-ci la coupe finit par retourner à Thalès, qui décide alors de la consacrer à Apollon, non pas à l'Apollon de Delphes, mais à l'Apollon de Milet : c'était ou bien l'Apollon du sanctuaire oraculaire de 
Didymes (à $16 \mathrm{~km}$ de Milet) ou bien l'Apollon Delphinios («Dauphin») de Milet. La version de Diogène Laërce est assez confuse, et il est oiseux de prétendre trancher le problème. L'essentiel est de voir que le бọóc, dans tous les cas, signale que le premier

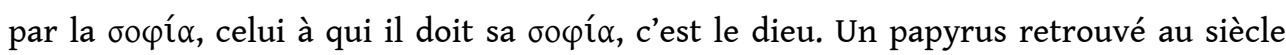
dernier nous a conservé le passage du poème de Callimaque où le fils de Bathyclès porte la coupe à Thalès. Où le trouve-t-il ? Dans le temple d'Apollon à Didymes. Et qu'est-il en train de faire ? Il trace des figures géométriques sur le sable ${ }^{58}$ :

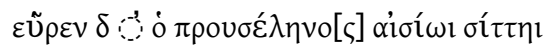

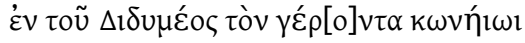

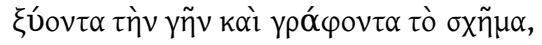

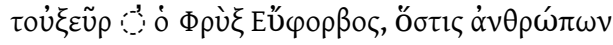

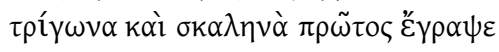

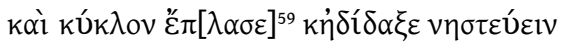

$\tau \tilde{\omega} v \dot{\varepsilon} \mu \pi v \varepsilon \operatorname{cov} \tau \omega \nu$.

L'homme-d'avant-la-lune ${ }^{60}$ le découvrit grâce à la sittelle de bon augure : le vieil homme était dans <le sanctuaire> du Didyméen. Il raclait la terre avec un bâton et dessinait la forme que trouva le Phrygien Euphorbe ${ }^{61}$, celui qui, le premier parmi les hommes, dessina des triangles, scalènes ou non, construisit un cercle et enseigna à s'abstenir des êtres qui respirent.

On ne saurait plus clairement inscrire l'exercice de la oopía, et l'exercice pour nous le plus « rationnel » de la бọía (faire de la géométrie), dans l'espace des dieux. Une autre histoire le confirme : lorsque Thalès parvint à inscrire un triangle rectangle dans un cercle, que fit-il aussitôt? Il sacrifia un bœuf ${ }^{62}$. Sans qu'on puisse l'affirmer, il est vraisemblable qu'il le sacrifia à Apollon, «seigneur de Lycie, de Méonie (Lydie) et de Milet $»^{63}$.

On ne sait pas à quand remontent ces histoires. Elles apparaissent à l'époque hellénistique, mais certaines sources (Théophraste, Eudoxe de Cnide) peuvent faire penser qu'elles sont plus anciennes. L'important, pour notre propos, c'est qu'elles nous montrent que, pour les Anciens, la sagesse de Thalès dépendait d'Apollon.

\section{Thalès voit des dieux partout}

Fort de cette relation avec Apollon, Thalès se comporte exactement comme un sage. D'abord il voit ce qui est caché dans les choses qui nous entourent. Il voit notamment que les choses en apparence inanimées sont en réalité animées ${ }^{64}$ :

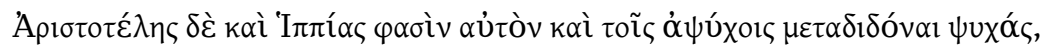

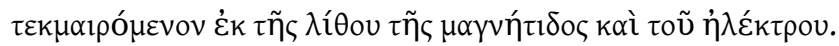

Selon Aristote et selon Hippias, Thalès attribuait une âme y compris aux choses inanimées. Il l'avait conjecturé à partir de la pierre de Magnésie et de l'ambre.

Le texte d'Hippias et d'Aristote cité par Diogène Laërce nous dit que Thalès a fait une

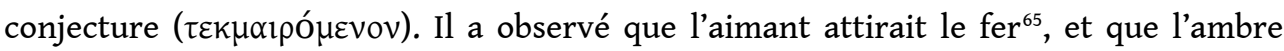
frottée attirait les corps légers (la paille, par exemple). L'acte d'observer, que nous aurions tendance, "spontanément", étant donné notre formation, à interpréter comme un acte précurseur de la modernité scientifique, est en réalité un acte

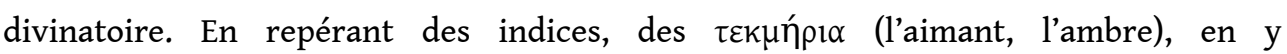

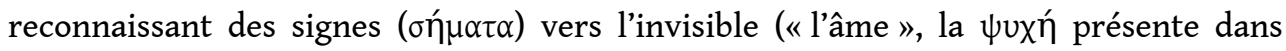
l'apparemment inanimé), Thalès se comporte comme les devins qui doivent eux aussi reconnaître les signes qu'Apollon dissémine pour celui qui sait voir. Marcel Detienne a consacré à cette "sémiologie» des devins des pages essentielles de son livre sur 
Apollon $^{66}$. Thalès a " repéré » que l'ambre frotté attirait la paille, que l'aimant attirait le fer, et il a vu là un signe envoyé par Apollon, de même que Théoclymène, dans l'Odyssée $(\mathrm{XV}, 525-534)$, voyait dans un aigle le signe que la famille d'Ulysse régnerait pour toujours sur Ithaque ${ }^{67}$.

En outre, Thalès n'a pas seulement vu, à partir de l'aimant et de l'ambre, que tout était plein d'âmes. Il a encore vu que ces âmes étaient des dieux, ce qui nous éloigne encore davantage du savoir empirico-déductif moderne :

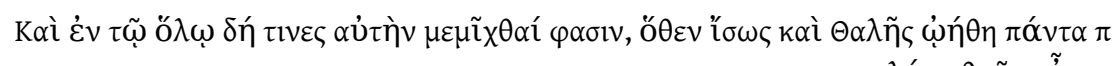

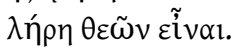
Certains disent que l'âme est mêlée dans tout l'univers : c'est peut-être pour cette raison que Thalès disait que tout est plein de dieux ${ }^{68}$.

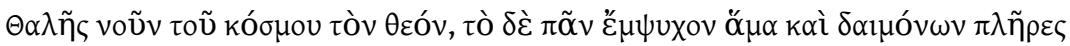

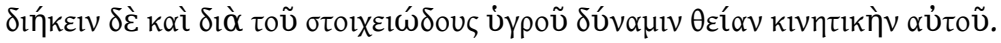

D'après Thalès, le voũ cst le dieu de l'univers, et le tout est animé et en même temps plein de divinités. Au moyen de l'humidité élémentaire, une puissance divine le parcourt et le met en mouvement ${ }^{69}$.

Mais comment se représente-t-il ces dieux ? On ne sait pas. Les témoignages parlent

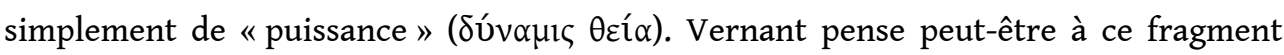
quand il dit que les dieux des Milésiens sont des abstractions, autrement dit que ce ne sont plus les dieux «anthropomorphes » des mythes ${ }^{70}$. Or nous ne pouvons pas savoir sous quelles formes Thalès se représentait ces "puissances", et rien ne permet d'exclure que ces formes aient été concrètes. En outre, nous allons voir, d'une part, qu'il n'y a aucune raison de donner au "mythe» une telle importance dans la description de la religion antique; et que, d'autre part, dans les cultes antiques, l'anthropomorphisme est compatible avec l'abstraction.

\section{Les prédictions de Thalès}

Par ailleurs, Thalès fait des prédictions : il voit l'avenir que nous ne voyons pas. Tantôt il voit l'avenir par intuition directe : c'est ainsi que Thalès voit, par exemple, que ce qui est de son vivant un "terrain vague » auquel personne ne fait attention (

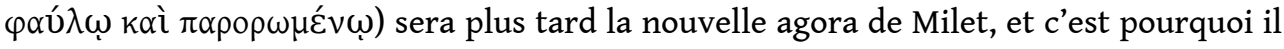
demande à y être enterré ${ }^{71}$. Tantôt il voit l'avenir comme il avait vu les " âmes » : par conjecture. En témoigne une anecdote amusante racontée par Aristote $^{72}$. On reprochait à Thalès d'être pauvre, et on en concluait que la philosophie ne servait à rien. Alors, pour montrer qu'avec son savoir, il pourrait parfaitement s'enrichir, mais qu'il s'en abstenait parce que l'argent ne l'intéressait pas, il observa les astres pendant l'hiver, et il en conclut que l'année suivante il y aurait une récolte d'olives très abondante. Il décide donc de louer à très bon marché tous les pressoirs à olive de la région de Milet et de Chios. L'année suivante, la récolte est très abondante, tout le monde lui réclame ses pressoirs. Comme il en détient le monopole, il fait fortune ${ }^{73}$. Là aussi, une lecture "spontanée ", c'est-à-dire configurée par toute notre formation scolaire et académique, nous conduirait à reconnaître dans cette observation des astres une attitude «déjà » scientifique. Or il est scientifiquement impossible de prévoir par l'observation des astres si l'année qui vient sera propice ou non à la récolte des olives. Là encore, il ne faut pas se laisser induire en erreur : il y a bien conjecture, mais cette conjecture n'a rien de scientifique. Elle résulte d'une vision de ce qui est invisible aux autres hommes. 
34 La plus connue des prédictions de Thalès, c'est bien sûr celle d'une éclipse de soleil. C'est vraisemblablement à cette occasion qu'il fut nommé sage ${ }^{74}$. Dans deux pages remarquables (et assez drôles) de son Ursprungsmythos der Vernunft - Zur philosophiehistorischen Genealogie des griechischen Wunders, Helmut Heit montre à quel point, contre toute rigueur scientifique, les savants ont salué cette prédiction comme l'avènement de la philosophie et de la science rationnelle ${ }^{75}$. Or, comme le savent bien les historiens de l'astronomie, notamment de l'astronomie babylonienne, la plus avancée à l'époque de Thalès, prédire une éclipse de soleil sur des bases scientifiques était à cette époque impossible ${ }^{76}$. Du reste, les textes ne nous disent rien de tel. Thalès a simplement « prédit » qu'il y aurait une éclipse : il a vu, grâce à Apollon, qu'il y aurait une éclipse.

\section{Thalès et le culte d'Apollon à Milet}

Jusqu'ici, j'ai examiné les témoignages dont nous disposons sur la бọía de Thalès. Je vais à présent adopter une démarche différente : plutôt que de partir des témoignages sur Thalès, je partirai des témoignages concernant le culte d'Apollon à Milet et montrerai qu'il est possible d'établir des liens entre ce culte et ce que nous savons de Thalès.

36 La ville de Milet était reliée au sanctuaire oraculaire d'Apollon à Didyme par une voie sacrée. À l'époque de Thalès, cette voie sacrée n'était pas encore pavée, et le temple était encore une structure en bois ${ }^{77}$, mais, malgré ces apparences modestes, c'était un oracle aussi important que celui de Delphes ${ }^{78}$, comme en témoignent les offrandes de Crésus $^{79}$ et celles du pharaon Néchao ${ }^{80}$.

Or nous avons une inscription qui décrit les rituels à accomplir pendant le parcours de cette voie sacrée par les Molpoï, c'est-à-dire des "chanteurs", des prêtres qui chantent ${ }^{81}$. Avant la procession, on installe une pierre au début et à la fin du parcours, donc à la porte sud de Milet et sur le seuil du temple. Ces deux pierres, nommées $\gamma \cup \lambda \lambda$ oí $^{82}$, sont sacrées ${ }^{83}$. Entre ces deux pierres, le parcours est scandé par des étapes, où les Molpoï font des sacrifices et adressent des chants à différentes divinités : Hécate $^{84}$, Hermès, les Nymphes, des héros ${ }^{85}$. Parmi ces divinités qui scandent le parcours, il y en a une qui s'appelle «Puissance » $\Delta \Delta$ v́vauıc. C'est le même mot que chez

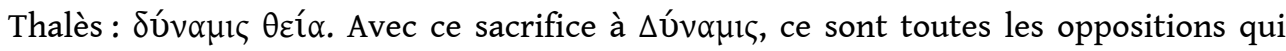
nous sont familières (abstrait/concret, concept/image), ce sont tous nos discours sur le supposé passage d'une religion anthropomorphique, mythologique, rituelle, à une religion abstraite, spirituelle, rationnellement épurée du mythe, qui s'effondrent. C'est pourquoi les savants se sont donné beaucoup de mal pour " désamorcer » ce sacrifice à $\Delta$ v́vauıc. Comment concevoir qu'un culte grec archaïque, donc un culte forcément anthropomorphe, s'adresse à une abstraction? On a proposé d'y voir la personnification du "pouvoir des magistrats ", et Wilamowitz a été jusqu'à vouloir en

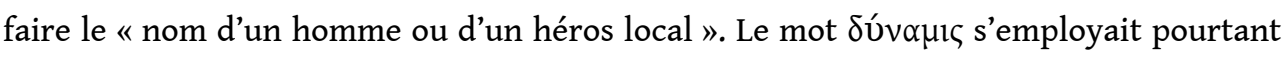
couramment pour décrire le pouvoir des dieux ${ }^{86}$. Les dieux sont ceux qui peuvent tout, et surtout l'impossible, comme on le lit sur une inscription d'Asie mineure ${ }^{87}$. On peut donc considérer $\Delta$ úvauı comme un équivalent de l'expression $\tau$ ò $\theta \varepsilon$ ĩov qu'on rencontre chez Anaximandre et sur de nombreuses inscriptions ${ }^{88}$.

Peut-on établir une relation, même hypothétique, entre ces Molpoï, ces prêtres chanteurs qui empruntaient cette voie sacrée, qui montaient sur cette hauteur, qui 
traversaient ces bois, qui chantaient devant ces sources, et ce sage qui disait que tout

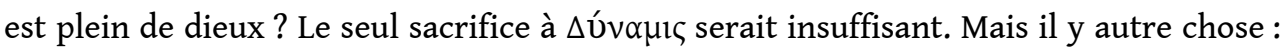
Louis Robert a rappelé à plusieurs reprises l'importance de la configuration actuelle des sites pour comprendre ce qui s'y passait pendant l'Antiquité ${ }^{89}$. On peut en dire autant, me semble-t-il, de ce que les gens racontent à propos de ces sites, de l'imaginaire qui les investit aujourd'hui encore. Or il se trouve que la hauteur qui autrefois servait d'étape à la procession des Molpoï est désignée aujourd'hui comme to psuchiko $0^{90}$, c'est-à-dire le lieu où il y a de l'âme, où il y a du Wuxikóv. On peut évidemment rejeter comme purement fortuite cette coïncidence, mais on peut aussi se demander si l'intuition

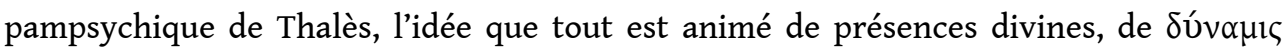
$\theta \varepsilon i ́ \alpha$, n'avait pas jadis un rapport avec cet espace parcouru par les Molpoï, année après année, entre Milet et Didymes.

\section{Anaxagore}

39 Anaxagore est considéré dans l'historiographie courante comme celui qui fait le lien entre ce qu'on appelle "l'école d'Ionie » et Athènes ${ }^{91}$. Il occupe donc une place très importante dans cette historiographie. On lui attribue la doctrine selon laquelle l'univers aurait été ordonné par le Noṽ «Intellect ». Cette traduction, qui éveille tout de suite des connotations qui nous sont familières, fait d'Anaxagore un homme des Lumières, un Aufklärer ${ }^{92}$. Et ce d'autant plus qu'il fut obligé de fuir Athènes, dit-on, parce qu'on l'aurait accusé d' " athéisme ».

Je vais donc là aussi, comme pour Thalès, essayer de montrer, à partir des témoignages

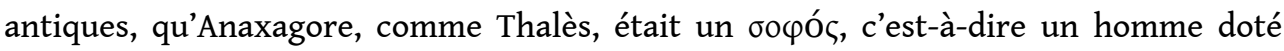
d'une capacité de vision qui lui venait des dieux.

\section{Les prédictions d'Anaxagore}

41 Anaxagore, comme Thalès, comme Anaximandre ${ }^{93}$, fait des prédictions : il prédit la chute d'une météorite à $\mathrm{Abydos}^{94}$, il prédit qu'il y aura la pluie aux jeux olympiques (contre toute attente) ${ }^{95}$; il prédit l'écroulement d'une maison, une éclipse de soleil, la submersion des montagnes de Lampsaque, des tremblements de terre (en remuant la boue d'un puits) ${ }^{96}$. Aucune de ces prédictions ne peut se faire de façon rationnelle, scientifique. Elles ne peuvent s'expliquer, pour les Anciens, que par la relation privilégiée d'Anaxagore avec les dieux: c'est un personnage aimé des dieux, un " homme divin », en un mot : un бoৎó $\varsigma^{97}$.

\section{Le voũs}

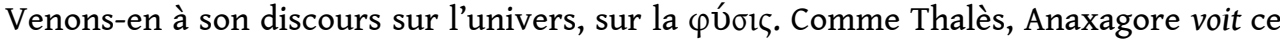
qui est caché : « ce qu'on voit n'est que l'apparence: des choses cachées $»^{98}$, comme dans

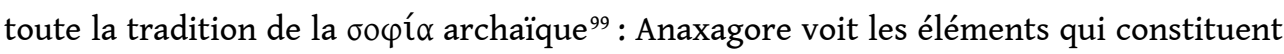
les choses (qu'il appelait "homéoméries » ${ }^{100}$ ), par exemple la chair, l'os, l'eau ou le $\mathrm{feu}^{101}$. Le reste des hommes ne voit que l'élément dominant ${ }^{102}$ : par exemple, dans l'eau, nous ne voyons que l'eau, mais nous ne voyons pas que l'eau contient aussi du bois, de l'écorce et du fruit ; nous ne voyons pas non plus que le pain contient de la chair, des os, des veines, des nerfs, des cheveux, des ongles, des ailes, des cornes, ni que les aliments contiennent du sang, ni que la pierre contient du feu, l'air des semences, la 
mer de l'air, etc. ${ }^{103}$. Dès lors, les apparences nous trompent : en réalité, tout est dans tout ${ }^{104}$. Pire encore : la faiblesse de nos sens ne nous permet même pas de percevoir correctement les apparences ${ }^{105}$ : ainsi la vraie couleur de la neige est le noir, c'est à tort que nous la percevons comme «blanche $»^{106}$.

Derrière ce désordre apparent des choses, où tout est dans tout, il y a un ordonnancement auquel préside le voṽ $\varsigma^{107}$. Mais qu'est-ce que c'est que ce voũ $\zeta$ ? La traduction de ce mot est d'autant plus difficile que, dans les témoignages hétérogènes et parfois contradictoires qui en font mention, il est parfois interchangeable avec $\psi v \times \eta ́$ ${ }^{108}$; c'est une puissance faite d'intelligence, de volonté et d'affect ${ }^{109}$. Il serait vain de prétendre donner une traduction définitive de ce mot. Une chose en tout cas est certaine, et décisive pour mon propos : le voṽ $\varsigma$ est un dieu ${ }^{110}$.

Comment Anaxagore a-t-il eu connaissance de ce voũ $\zeta$ ? Est-ce par intuition directe, est-ce par conjecture, comme Thalès avec la pierre? Aristote nous apporte ici un témoignage fondamental ${ }^{111}$ :

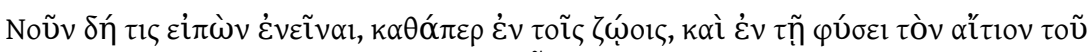

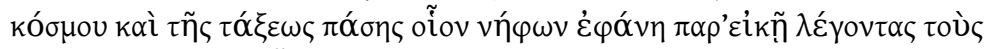

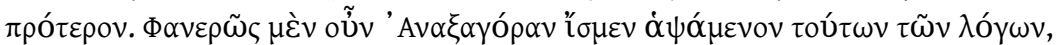

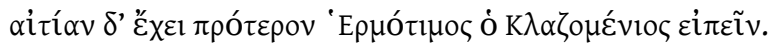

Quelqu'un ayant dit qu'il y a du voũ dans l'univers au même titre que dans les vivants, et que c'est la cause de l'univers et de tout son ordonnancement, apparut comme un homme sensé, à côté de ceux d'avant, qui discouraient à l'aventure. Nous savons bien qu'Anaxagore a tenu ces discours, mais ils remontent à Hermotime de Clazomènes.

Donc, Anaxagore a un précurseur en la personne d'un concitoyen, Hermotime. Qui est cet Hermotime? Un homme qui s'était rendu célèbre par la capacité qu'avait son âme de quitter son corps. Pendant que son corps gisait inanimé, son âme « errait au loin » et "voyageait de nombreuses années en différents lieux $»^{112}$. À son retour, son âme

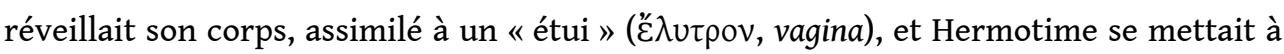
raconter « des choses qui s'étaient passées loin de sa cité, que seul un témoin avait pu connaître ", ou encore prédisait l'avenir ${ }^{113}$. Le souvenir d'Hermotime s'était conservé, dans l'école de Platon ${ }^{114}$, mais aussi dans l'école d'Aristote, comme le montre, outre le texte que nous venons de citer, ce fragment de Cléarque de Soles, un des disciples d'Aristote ${ }^{115}$ :

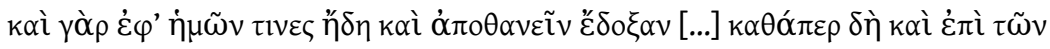

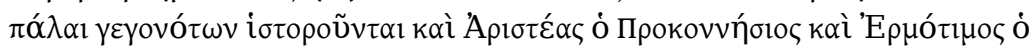

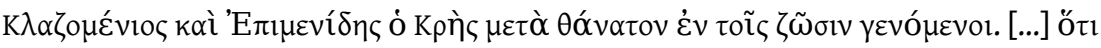

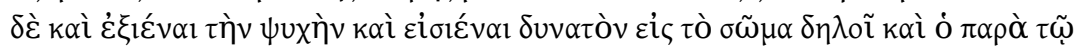

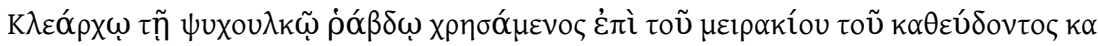

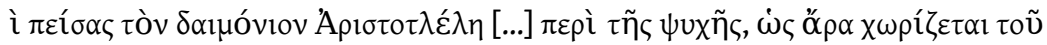

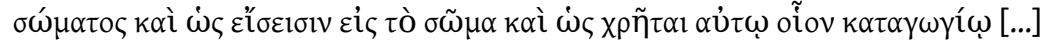

D'ailleurs, de notre temps, nous avons vu des gens qui n'étaient morts qu'en apparence [...]. De même, parmi les Anciens, Aristée de Proconnèse, Hermotime de Clazomènes et Épiménide le Crétois rapportent qu'ils sont morts puis qu'ils sont revenus parmi les vivants. [...] Mais que l'âme est capable de sortir du corps et d'y rentrer, cela a été démontré par celui qui appliqua une baguette " attire-l'âme » à l'adolescent qui dormait, et qui convainquit le divin Aristote [...] que l'âme se sépare du corps, qu'elle y retourne, et qu'elle en use comme d'une auberge [...] encore leurs deux noms dans son Protreptique, et là aussi à propos du voṽ $\varsigma^{116}$ : 


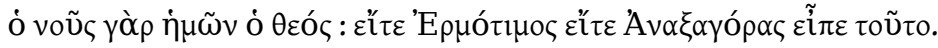

Notre voũc est le dieu. Hermotime ou Anaxagore l'a dit. l'insolente Athènes, Anaxagore avait fini par être assimilé à un "professionnel du

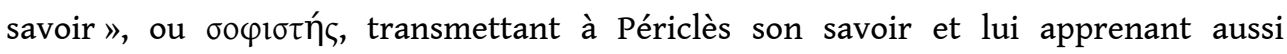
l'éloquence ${ }^{128}$. Nous le voyons, dans une anecdote, traité par Périclès avec une désinvolture qui rappelle celle de Calliclès à l'égard de Gorgias ${ }^{129}$. Et pourtant,

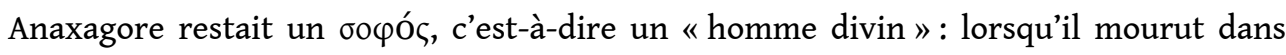
son exil de Lampsaque, les habitants le célébrèrent aussitôt comme un dieu ${ }^{130}$ et lui élevèrent un autel encore visible à l'époque d'Élien, dédié au Noṽ s et à la Vérité ${ }^{131}$, selon une pratique religieuse courante dans le monde grec, consistant à reconnaître et à honorer comme dieux des personnages qui avaient manifestement été aimés par les dieux, qu'il s'agisse d'athlètes, de poètes ou de sages ${ }^{132}$. Sur le même autel ou sur un 
autre, on lisait une autre inscription: "Ci-gît Anaxagore, qui a plus qu'aucun autre approché du terme de la Vérité $»^{133}$. Ces inscriptions sont peut-être des inventions, mais la réalité du culte ne fait pas de doute. Comme Alcidamas cite le culte d'Anaxagore à Lampsaque en même temps que celui du poète Archiloque à Paros ${ }^{134}$, on peut penser

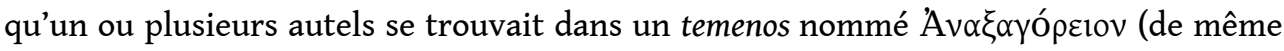
qu'il y avait un Apxı $\lambda o ́ x \varepsilon 10 v$ à Paros) et que, de même qu'à Paros on « dressait plusieurs

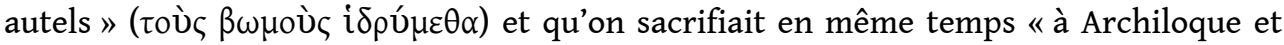

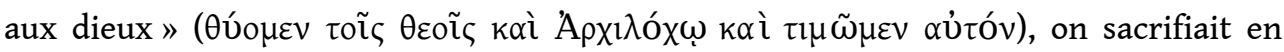
même temps à Anaxagore et "aux dieux " (peut-être, si on en juge l'inscription, au Noũ et à la Vérité).

52 Anaxagore avait demandé qu'on laisse les enfants jouer chaque année, pour le mois anniversaire de sa mort, et la coutume durait encore à l'époque de Diogène Laërce ${ }^{135}$. Quel est le sens de cette coutume ? Elle évoque l'histoire d'Héraclite jouant aux osselets avec les enfants dans le temple d'Artémis à Éphèse, au lieu de participer à la vie politique $^{136}$. Anaxagore aussi, dit-on, se voyait accuser par ses concitoyens de ne pas s'occuper de politique parce qu'il ne faisait que contempler les astres ${ }^{137}$. À l'un d'entre eux qui lui en faisait reproche, il répondit: "Tais-toi, de ma patrie, j'ai souci », et il

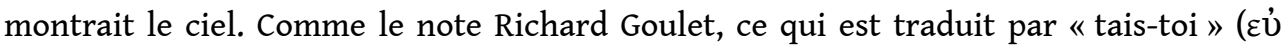
$\varphi \eta \mu \varepsilon \tilde{)})$ est une «injonction à connotation religieuse ${ }^{138}$, recommandant de garder le silence pour éviter de prononcer des paroles blasphématoires ». On voit ici comment une anecdote sans doute fictive peut nous éclairer sur un rituel effectivement pratiqué, nous donnant des informations précieuses sur une conception de la бopía encore active sous l'Empire à l'époque des Sévères.

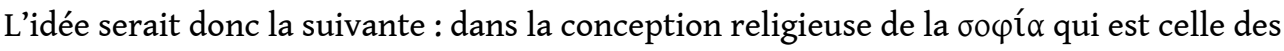
sages d'Ionie et que l'on retrouve chez Aristote ${ }^{139}$, le sage, proche des dieux, ne s'occupe pas de politique : les affaires humaines ne sont pas sérieuses. C'est pourquoi le sage se sent plus proche des enfants qui jouent que des hommes qui se préoccupent de la Cité. Car il s'agit au moins d'une activité qui ne prétend pas à tort être sérieuse, et qui en plus nous rapproche des dieux ${ }^{140}$.

J'espère avoir montré que le savoir des Ioniens était fondé sur une relation du sophos avec les dieux. Les dieux ne sont pas critiqués. Ils ne sont pas davantage tenus «en dehors» des pratiques de la sophia. Cette relation du sophos aux dieux, nous la retrouverons dans toute l'histoire de la philosophie antique : chez Socrate, chez Platon, chez Antisthène, chez Aristote, chez Épicure, chez les Stoïciens. Les écoles athéniennes, rappelons-le, étaient des associations constituées autour de cultes rendus aux dieux. La question que les antiquisants, dès lors, doivent affronter, est la suivante : comment concilier le caractère incontestablement rationnel de certains savoirs et de certaines pratiques des philosophes antiques ${ }^{141}$ avec leur relation privilégiée aux dieux? Les tentatives de réponses à cette question gagneront beaucoup à sortir l'histoire des savoirs antiques de son isolement et à dialoguer, de manière comparatiste, avec les recherches des historiens du Moyen Âge et des historiens modernes sur les rapports entre « science » et « religion». 


\section{BIBLIOGRAPHY}

P. ADAMSON, Classical Philosophy: A history of philosophy without any gaps, Oxford, 2014.

J. BARNES, « Greek Wisdom ii », CR 29 (1979), p. 242-243.

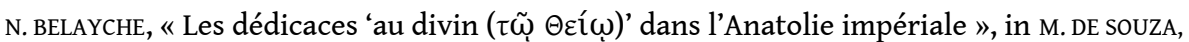
A. PETERS-CUSTOT, F.-X. ROMANACCE (éd.), Le sacré dans tous ses états. Catégories du vocabulaire religieux et sociétés, de l'Antiquité, nos jours, Saint-Étienne, 2012, p. 181-194.

C. BLACKWELL, «Thales philosophus: The Beginning of Philosophy as a Discipline », in D.R. KELLEY

(éd.),History and the Disciplines. The Reclassification of Knowledge in Early Modern Europe, Rochester (NY), 1997, p. 61-82.

H. BLUMENBERG, Le rire de la servante thrace, tr. fr., Paris, 2000.

C. BONNET, « Mise en perspective épistolaire. 'Denn ich denke oft im Stillen an Sie'. Hermann Diels et Franz Cumont : la filiation intellectuelle à l'épreuve de la guerre 14-18 », Anabases 10 (2009), p. 99-110.

P. BORGEAUD, Recherches sur le dieu Pan, Genève, 1979.

P. BOURDIEU, Méditations pascaliennes, Paris, 1997.

P. BOYANCÉ, Le Culte des Muses chez les philosophes grecs. Études d'histoire et de psychologie religieuse, Paris, $1972^{2}$ [1937].

S. BROADIE, « Rational Theology », in A.A. LONG(éd.),The Cambridge Companion to Early Greek Philosophy, Cambridge, 2006, p. 205-224.

W. BURKERT, Lore and Science in Ancient Pythagoreanism, Cambridge [Mass.]), $1972^{2}$ [1961].

R.G.A. BuXTon(éd.), From Myth to Reason? Studies in the Development of Greek Thought, Oxford, 1999.

C. CALAME, «The Rhetoric of Muthos and Logos: Forms of Figurative Discourse », in BUXTON (1999), p. 119-143.

_, Mythe et histoire dans l'antiquité grecque. La création symbolique d'une colonie, Paris $2011^{2}$ [1996].

-, « Mûthos et lógos : les pouvoirs du discours dans deux tragédies d'Eschyle », in M. BROzE, B. DECHARNEUX, S. DELCOMinetTE (éd.), « All'eû moi katálexas. Mais raconte-moi en détail... », Mélanges de philosophie et de philologie offerts à Lambros Couloubaritsis, Bruxelles/Paris, 2008, p. 179-194.

-, « Procédures hymniques dans les vers des sages cosmologues. Pragmatique de la poésie didactique (d'Hésiode et Théognis à Empédocle et Parménide) ", in R. BOUCHON, P. BRILLET-DUBOIS, N. LE MEUR-WEISSMAN (éd.), Hymnes de la Grèce antique. Approches littéraires et historiques. Actes du colloque international de Lyon, 19-21 juin 2008, Lyon, 2013, p. 59-77.

-, Qu'est-ce que la mythologie grecque?, Paris, 2015.

-, « Les Rédacteurs grecs d'enquêtes sur le passé héroïque : ni mythographes, ni mythographie », Kernos 29 (2016), p. 403-414.

-, « À propos des pratiques divinatoires et de la raison des signes en Grèce classique », Mètis n.s. 15 (2017, sous presse).

L. CANFORA, 1914, Palerme, 1992 
J.-M. CARBON, « Dolphin-Pillars », EA 46 (2013), p. 27-34.

C. CASTORIADIS, Ce qui fait la Grèce 1. D’Homère à Héraclite, Paris, 2004.

D. CLAY, Archilochos Heros: The Cult of Poets in the Greek Polis, Cambridge (Mass.), 2004.

J.S. CLAY, « The Planktai and the Moly: Divine Naming and Knowing in Homer », Hermes 100 (1972), p. $127-131$.

G. colli, La nascita della filosofia, Milan, 1975.

-, La Sapienza greca, t. I : Dioniso. Apollo. Eleusi. Orfeo. Museo. Iperborei. Enigma, Milan, 1977 ; t. II : Epimenide. Ferecide. Talete. Anassimandro. Anassimene. Onomacrito, Milan, 1978 ; t. III : Eraclito, Milan, 1980.

F.M. CORNFORD, From religion to philosophy: a study in the origins of western speculation, Londres, 1912.

_, Principium sapientiae. The Origins of Greek Philosophical Thought, Oxford, 1952.

P. CURD, Anaxagoras of Clazomenae: Fragments and Testimonia. A text and translation with notes and essays, Toronto, 2007.

B. CURRIE, Pindar and the Cult of Heroes, Oxford, 2005.

M.-L. DESCLOS, F. FRONTEROTTA, «Introduction », in M.-L. DESCLOS, F. FRONTEROTTA (éd.), La Sagesse présocratique - Communication des savoirs en Grèce archaïque : des lieux et des hommes, Paris, 2013, p. 7-20.

M. DETIENNE, Apollon le couteau à la main, Paris, 1998.

—, « En ouverture. Retour sur la bouche de la Vérité », in DETIENNE (2005), p. 89-111.

—, Les Maîtres de Vérité dans la Grèce archaïque, 2006² [1967].

-, Les Dieux d'Orphée, Paris, $2007^{2}$ [1989].

_, Les Grecs et nous. Une anthropologie comparée de la Grèce ancienne, Paris, 2005.

-, Comparer l'incomparable, Paris, $2009^{2}$ [2000].

H. DIELS, « Anaximandros von Milet », Neue Jahrbücher für dasKlassische Altertum 51 (1923), p. 65-76.

-, Die Fragmente der Vorsokratiker (1903), 6 éd. révisée par W. Kranz, Berlin, 1951-1952.

E. R. DODDS, Les Grecs et l'irrationnel, tr. fr., Paris, 1977 [1959].

T. DORANDI, «Le Traité sur le sommeil de Cléarque de Soles : catalepsie et l'immortalité de l'âme », ExClass 10 (2006), p. 31-52.

P. DUHEM, Sauver les apparences. Sur la notion de théorie physique de Platon à Galilée, Paris, 2008 [1908].

P.J. VAN DER EIJK, « The Role of Medicine in the Formation of Early Greek Thought », in P. CURD,

D.W. GRAHAm (éd.), The Oxford Handbook of Presocratic Philosophy, Oxford, 2008, p. 385-412.

L.R. FARNELL, Greek Hero Cults and Ideas of Immortality, Oxford, 1921.

J. E. FONTENRose, Didyma: Apollo's Oracle, Cult and Companions, Berkeley, 1988.

G. FOWDEN, The Egyptian Hermes: A Historical Approach to the Late Pagan Mind, Princeton, $1993^{2}$ [1986].

R.L. FOWLER, « Mythos and logos »JHS 131 (2011), p. 45-66.

S. GEORGOUDI, « La procession chantante des Molpes de Milet », in P. BRULÉ, C. VENDRIES (éd.),

Chanter les dieux. Musique et religion dans l'Antiquité grecque et romaine, Rennes, 2001, p. 153-170. 
S. GÖDDE, Euphemia. Die gute Rede in Kult und Littteratur der griechischen Antike, Heidelberg, 2011.

K.B. GÖDECKEN, « Beobachtungen und Funde an der Heiligen Strasse zwischen Milet und Didyma, 1984 », ZPE 66 (1986), p. 217-253.

V.B. GORMAN, Miletos, the Ornament of Ionia: A History of the Ccity to 400 B.C.E, Ann Arbor (Mich.), 2001.

F. GRAF, « Das Kollegium der Molpoi von Olbia », MH 31 (1974), p. 2-22.

D.W. GRAHAM, Explaining the Cosmos: the Ionian Tradition of Scientific Philosophy, Princeton, 2006.

A.M. GREAVES, Miletos: A History, Londres/New York, 2002.

F.D. HARVEY, « Literacy in the Athenian Democracy », REG 79 (1966), p. 585-635.

H. HEIT, « Did Rationality Originate in Ancient Ionia? », Skepsis 15 (2004), p. 359-371.

-, Ursprungsmythos der Vernunft - Zur philosophiehistorischen Genealogie des griechischen Wunders, Würzburg, 2007, p. 102-114

A. HERDA, Der Apollon-Delphinios-Kult in Milet und die Neujahrsprozession nach Didyma. Ein neuer Kommentar der sog. Molpoi-Satzung, Mayence, 2006.

E. HUSSEY, « Ionian Inquiries: On Understanding the Presocratic Beginnings of Science », in A. POWELL(éd.),The Greek World, Londres, 1995, p. 530-549.

D. JANICAUD, Hegel et le destin de la Grèce, Paris, 1975.

R. KOCH-PIETTRE, Comment peut-on être dieu ? La secte d'Épicure, Paris, 2005.

A. LAKS, Introduction à la « philosophie présocratique », Paris, 2006.

-, G.W. MOST, Les débuts de la philosophie. Des premiers penseurs grecs à Socrate, Paris, 2016.

M.-C. LECLERC, La Parole chez Hésiode, Paris, 1993.

H. LEWY, « Aristotle and the Jewish Sage According to Clearchus of Soli », HThR 31 (1938), p. 205235.

G.E.R. LLOYD, Les débuts de la science grecque, tr. fr., Paris, 1974.

—, « Mythology: Reflections from a Chinese Perspective », inBUXтon (1999), p. 145-165.

C. MACRIS, « Pythagore de Samos », in R. GOULET, Dictionnaire des philosophes antiques, VII, Paris, 2018 (sous presse), p. 681-850.

J. MANSFELD, «The Chronology of Anaxagoras' Athenian Period and the Date of His Trial ", Mnemosyne 32 (1979), p. 3969.

M. MARCovich, « Herakleitos », RE Suppl. X (1965), col. 246-320.

G.W. MOST, «From Logos to Mythos »,in BUXTON (1999), p. 25-47.

W. NESTLE, Vom Mythos zum Logos: die Selbstentfaltung des griechischen Denkens von Homer bis auf die Sophistik und Sokrates, Stuttgart, 1940.

O.E. NEUGEBAUER, A History of Ancient Mathematical Astronomy, 3 vol., Berlin, 1975.

F. NIETZSCHE, Les Philosophes préplatoniciens, suivi de Les $\delta 1 \alpha \delta$ oxaí des philosophes, tr. fr., Combas, $1994[1872]$.

H.W. PARKE, « The Massacre of the Branchidae », JHS 105 (1985), p. 59-68.

G. PETZL, Die Beichtinschriften Westkleinasiens, Bonn, 1994 (Epigraphica Anatolica, 22). 
O. PRIMAVESI, « Empedokles », in D. BREMER, H. FLASHAR, G. RECHENAUER(éd.),Grundriss der Geschichte der Philosophie. Die Philosophie der Antike, I, Frühgriechische Philosophie, Bâle, 2013, p. 667-739.

L. ROBERT, « De Delphes à l'Oxus. Inscriptions grecques nouvelles de la Bactriane », CRAI 112

(1968), p. 416-457.

-, « Monnaies hellénistiques. I. Une monnaie de Rhodes contremarquée. II. L'argent d'Athènes stéphanéphore. Addition (monnaie d'Hypaipa) », RN 6 (1977), p. 7-47 [republié in ROBERT (1989) p. 169-209].

-, « Les conquêtes du dynaste lycien Arbinas », JS (1978), p. 21 [republié in ROBERT (1990), p. 381426].

-, Opera Minora Selecta VI, Paris, 1989.

-, Opera Minora Selecta VII, Paris, 1990.

H. SCHMID, «Sur la généalogie du rationnel : une ou deux questions », Philosophie antique 7 (2007), p. 39-50.

N. SWERDLOW, Otto E. Neugebauer, Washington, D.C., 1998.

G. THOMSON, « From Religion to Philosophy », JHS 73 (1953), p. 77-84.

C. vassallo, The Presocratics in the Herculaneum Papyri: Texts, Translations, and Commentary (= Studia Praesocratica), Berlin/Boston, De Gruyter (à paraître en 2019).

J.-P. VERNANT, « Du mythe à la raison. La formation de la pensée positive dans la Grèce archaïque », Annales (ESC) 12 (1957), p. 183-206 [republié in VERNANT (1985²), p. 373-403].

-, «Questions de méthode. Dialogue avec Maurice Caveing et Maurice Godelier », 1975 [republié in VERNANT (1996), p. 105-136].

-, Mythe et pensée chez les Grecs. Études de psychologie historique, Paris, 1985 [1965].

-, Entre mythe et politique, Paris, 1996.

A.-G. WERSINGER, « Présentation », RMM 75 (2012/2013), p. 291-299.

U. von WILAMOWITZ-MOELLENDORFF, Der Glaube der Hellenen, 2 vol., Berlin, 1931-1932.

K.W. YU, « From Mythos to Logos: Jean-Pierre Vernant, Max Weber, and the Narrative of Occidental Rationalization », Modern Intellectual History (2015), p. 1-30.

\section{NOTES}

1. Cf. par exemple BLACKWELL (1997). Pour une critique philosophique du raisonnement consistant à reconnaître comme « rationnel » la théorie de l'eau comme principe, cf. HEIT (2004). Il montre en effet que, quelle que soit la définition donnée d'un raisonnement « rationnel », il est dans tous les cas arbitraire de qualifier de « rationnelle » la proposition que l'eau est le principe de toutes choses.

2. LAKS (2006), p. 31-53.

3. Cf. JANiCAud (1975). Sur le Thalès de Hegel, cf. HeIt (2007), p. 102-114. Hegel n'est pas le premier à faire de Thalès le premier philosophe. L'idée apparaît pour la première fois au livre I de la Métaphysique d'Aristote (983b 20). Mais c'est l'interprétation qu'en donne Hegel qui est nouvelle et qui définit la doxa moderne. Pour l'Aristote de la Métaphysique, les " premiers à philosopher » (983b 6), ce sont ceux qui recherchent « les principes de toutes choses» (ibid.). Mais Hegel est le 
premier à donner à cette idée le sens d'un passage de la religion à la philosophie ou du mythe à la Raison.

4. Sur la relation de l'historiographie zellerienne à Hegel, cf. HEIT (2007), p. 29, 99-100.

5. Cf. NIETZSCHE (1994 [1872]), p. 110-111, notamment p. 107 : «Ce fut [sc. Thalès] un grand mathématicien, grâce à qui la philosophie commença en Grèce. De là vient son sens de l'abstrait, du non-mythique, du non-allégorique. » Renvoyons aussi aux pages sur Thalès dans La Philosophie à l'époque tragique des Grecs, trad. fr., Gallimard, Paris, 1975, p. 20-24.

6. Cf. BouRdieu (1997), p. 30 : «Le champ philosophique est sans nul doute le premier champ scolastique qui se soit constitué en s'autonomisant par rapport au champ politique en voie de constitution et par rapport au champ religieux, dans la Grèce du $\mathrm{v}^{\mathrm{e}}$ siècle avant notre ère ; et l'histoire de ce processus [...] est inséparable de l'histoire du processus qui a conduit de la raison analogique (celle du mythe et du rite) à la raison logique (celle de la philosophie). » Ce champ est "affranchi des prescriptions de la sagesse religieuse», et "les mythes et les rites s'y transforment d'actes pratiques de croyances [...] en objets d'étonnement et d'interrogation théoriques $[\ldots]$ ».

7. Cf. LAKS (2006), p. 131-149.

8. Cf. les textes auxquels renvoie LAKS (2006), p. 100-101.

9. Cf. son livre sur Le rire de la servante thrace, trad. fr., L'Arche, Paris, 2000.

10. Cf. CASTORIADIS (2004).

11. Cf. le parcours historiographique proposé par LAKS (2006), des Lumières à nos jours. Aux auteurs qu'il passe en revue, ajoutons les noms de François Châtelet, de Jean Bollack, de Pierre Hadot, de Marcel Conche.

12. VERNANT (1985 [1965]), p. 373-403.

13. CORNFORD (1912) ; ID. (1952).

14. D'après lui, la philosophie « rationalisait » le mythe, cf. CORNFORD (1952), p. 187-188.

15. VERNANT (1985 [1965]), p. 378. La notion de «mutation mentale» vient de la psychologie historique d'Ignace Meyerson, comme l'a montré LAKS (2006), p. 88-89.

16. Cf. l'interrogation de Maurice Caveing dans vernant (1996 [1975]), p. 113.

17. BUXTON (1999).

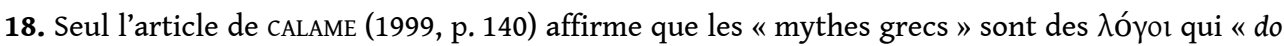
not seem to be markedly differentiated from other types of discourse in which we, by contrast, would perceive the operation of reason ". Cette critique de l'opposition muthos/logos a été développée ensuite par C. Calame dans de nombreux travaux, cf. CALAME $\left(2011^{2}\right.$ [1996]) ; ID. (2008) ; (2015) ; (2016) ; (2017), p. 23-76.

19. Sur ce " mariage de la carpe et du lapin », cf. EIJK (2008), p. 393 : "There is now a much greater willingness among interpreters to accept the religious and rational elements as coexistent and -at least in their author's conception - compatible » (je souligne); DESCLOS - FRONTEROTTA (2013), p. 13 : «Ce qui relève du religieux et ce qui relève du rationnel peuvent fort bien marcher du mê.ime pas sans s'exclure l'un l'autre.»

20. De même, LLOYD (1999) s'emploie à montrer, par une comparaison entre la Chine et la Grèce, que seuls les Grecs distinguaient entre «rationnel » et «imaginaire», et connaissaient la pratique du débat public.

21. NESTLE (1940), p.13: "Ein Wort wie das Luthers von der 'blinden Hure Vernunft' wäre im griechischen Kulturkreis unmöglich. Es konnte nur fallen vermöge der Unterordnung unter die jüdische Anschauung, dass das menschliche Erkenntnisstreben eine Auflehnung gegen Gott sei

22. MOST (1999), p. 30 : «Nestle was apparently not at all a Nazi himself and seems to have thought, oddly, thatby means of his book he could help to increase the rationality in the troubled worldaround him. »

23. LAKS (2006), p. 57, n. 2. 
24. «La formule du 'mythe à la raison' a été souillée par W. Nestle», mais «l'émergence et le développement de la rationalité en Grèce comme une sortie hors du mythe [...] ne pose aucun problème particulier » (ibid., p. 57).

25. La philosophie, pour Zeller (cité par HEIT [2007], p. 131), est « ein einheimisches Erzeugnis aus dem Geist und den Bildungszuständen des griechischen Volkes ».

26. FOWLER (2011). Cf. aussi GRAHAM (2006). Pour d'autres exemples, cf. HeIT (2004), p. 359.

27. Sur la « vive obsession » du muthos et du logos, cf. DETIENNE (2009² [2000]), p. 134-135, 145. Sur le retour de Vernant au mythe du miracle grec, cf. DETIENNE, ibid., p. 150-152.

28. DETIENNE (2005), p. 92 : «Comment se fait le passage d'une pensée marquée par l'ambiguïté et par sa logique à une autre qui semble ouvrir un nouveau régime intellectuel, celui de l'argumentation, du principe de non-contradiction, ainsi que du dialogue avec le sens, avec l'objet d'un énoncé et sa référence? » Plus bas (p. 106), il explique que dans son Invention de la mythologie, il n'avait nullement pour intention d'éliminer la catégorie du mythe, mais simplement de la « repenser ». Cf. aussi ce qu'il dit du logos (p. 93) : "Par sa fonction nouvelle et qui est fondamentalement politique, en accord avec l'agora, le logos, parole et langage, devient un objet autonome, soumis à ses propres lois. »

29. DETIENNE (1998), p. 10-11.

30. Car on ne peut soupçonner M. Detienne d'ignorer le problème, cf. e.g. DETIENNE (2009² [2000]), p. 134-135. De la même façon, on peut se demander, avec André LAKS (2006, p. 100-103), pourquoi Max Weber ne s'est jamais « attaqué » au problème du rationalisme grec. Craignait-il de devoir renoncer au mythe de la Raison grecque? Alors qu'il met en place un gigantesque chantier comparatiste, dans lequel il confronte les différents types de rationalité présents dans les différentes civilisations, Weber "n'a jamais traité du rationalisme grec pour lui-même ", " même s'il rend hommage ici ou là au rationalisme grec ». Le " même si » doit peut-être être remplacé par un "parce que ». Si "la Grèce joue chez Weber un rôle plus discret [...] que l'on ne le souhaiterait, au vu de l'importance même qu'il lui reconnaît dans la formation du rationalisme moderne... » (ibid., p. 103), c'est justement en raison de cette importance : un lecteur de Nietzsche comme Weber soupçonnait sans doute qu'une enquête véritable révélerait que le « rationalisme grec » n'était pas «pour l'essentiel extra-religieux» (ibid., p. 102). Je voudrais signaler maintenant également les réflexions de Kenneth W. YU (2015) sur Weber, Vernant, et «the Narrative of Occidental Rationalization ».

31. Le livre de W. Burkert sur Pythagore a fait définitivement justice du mythe d'un Pythagore mystique doublé d'un rationaliste inventeur des mathématiques. En ce qui concerne Empédocle, la plupart des chercheurs ont abandonné l'idée d'une opposition entre un Empédocle « rationnel », auteur du Sur la nature, et d'un Empédocle mystique, auteur des Purifications. Cf. en dernier lieu le texte remarquablement documenté d'Oliver PRIMAVESI (2013), en particulier p. 685694, ainsi que les réflexions d'Anne-Gabrièle WERSINGER (2012/2013), notamment p. 293.

32. DIELS (1923), p. 65. C'est le texte d'une conférence prononcée au Danemark et en Suède au printemps 1922.

33. CANFORA (1992), p. 109.

34. BONNET (2009).

35. DODDS (1977 [1959]), p. 139-178.

36. Quand Colli n'était pas ignoré, il était traité avec une désinvolture incroyable : en témoigne par exemple le compte rendu étique accordé par Jonathan BARNES au volume II de la Sapienza greca. Dans l' " orientation bibliographique » de l'édition Laks-Most, à la rubrique "Éditions et traductions partielles des premiers philosophes grecs ", l'œuvre de Colli n'est pas mentionnée. Il s'agit pourtant d'un instrument de travail indispensable, ne serait-ce que par l'apparat critique et les renvois bibliographiques qui accompagnent chaque fragment. J'ai signalé cet oubli aux deux éditeurs et espère qu'il sera réparé dans les prochaines éditions. 
37. BUXTON (1999), p. 11 (l'auteur souligne).

38. Nous disposons de trop peu de témoignages sur la vie d'Anaximandre et d'Anaximène, « intermédiaires» entre Thalès et Anaxagore, pour que l'on puisse, sans reconstructions arbitraires, les prendre pour objet d'étude. Les sources les concernant sont presque toutes uniquement doxographiques.

39. Sur le séjour d'Anaxagore à Athènes, nous avons deux traditions : l'une dit qu'il serait venu au lendemain de la deuxième guerre médique, l'autre qu'il y aurait philosophé trente ans. Comme il est mort en 428 , peu après son procès et son départ d'Athènes, les deux traditions ne sont pas conciliables et il est vain de prétendre trancher en faveur de l'une ou de l'autre.

40. L'expression, reprise par J.-P. VERNANT (1985 [1965]), p. 408, parfois attribuée à Lloyd, vient en fait de B.Farrington, cité par LloYd (1974), p. 20. On retrouve l'idée par exemple dans la présentation que l'éditeur fait du volume collectif La pensée antique des fondateurs à Saint Augustin, Paris, 2006 : «Depuis que Thalès, au vi ${ }^{\mathrm{e}}$ siècle avant Jésus-Christ, a commencé à chercher loin des dieux les réponses aux grandes questions du monde, c'est auprès des philosophes grecs que nous avons appris à nous interroger et à développer notre capacité à vivre en harmonie avec le monde.»

41. C'était peut-être son parent, Souda, s.v. ; Diogène Laërce, II, 1.

42. Diogène Laërce, I, 22.

43. I, 170. Sur le commerce entre Milet et le Proche Orient, cf. GREAVES (2002), p. 99. Sur Milet, on peut également consulter GORMAN (2001).

44. Pace THOMSON (1953), p. 79.

45. Hérodote, $V, 28$.

46. GREAVES (2002), p. 96.

47. Pline l'Ancien, V, 112. Cf. GREAVES (2002), p. 82 et 104.

48. Alcée, fr. 448 Lobel-Page (Himérius, Excerpt. Neap. Or., 28, 2 Colonna) = Thalès, 11 A 11a DK =

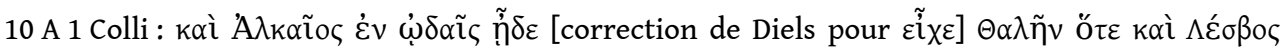
$\pi \alpha v \eta ́ \gamma v \rho ı$ [le texte s'interrompt ici], «Et Alcée célébrait Thalès dans ses chants, lorsqu'il y eut une fête à Lesbos. » Ce témoignage, le plus ancien dont nous disposions sur Thalès, ne figure pas dans l'édition Laks-Most. Il me paraît excessivement sceptique de penser qu'il s'agit d'un autre Thalès : un autre sage, Aristodème de Sparte, était chanté par Alcée (fr. 360 Lobel-Page [Diogène

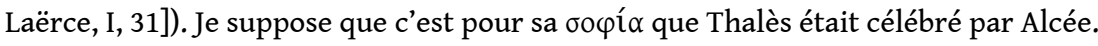

49. Diogène Laërce, I, 22 (= Démétrius de Phalère, fr. 149 Wehrli).

50. Il. I, 70; Hésiode, Theog., 38. Cf. les pages que Detienne $\left(2006^{2}\right.$ [1967]) a consacrées à cette бофí $\alpha$ divinatoire.

51. Cf. COLLI (1975), ch. 4.

52. Cf. Épiménide, 3 B 11 DK $=8$ A 4 Colli [Ce témoignage ne figure pas dans l'édition Laks-Most qui, contrairement à celle de Diels, ne consacre pas de chapitre spécifique à Épiménide. De la trentaine de témoignages sur Épiménide rassemblés par Diels, seuls deux ont survécu, dispersés dans deux chapitres de la section «Contexte et antécédents ». Le lecteur curieux de savoir qui était cet homme apprendra, dans l'index sélectif des noms propres, que c'était un "voyant et poète crétois légendaire" (c'est au contraire très vraisemblablement une figure historique), « réputé pour être resté endormi 57 ans dans une cave (sic) et s'être réveillé doué de pouvoirs

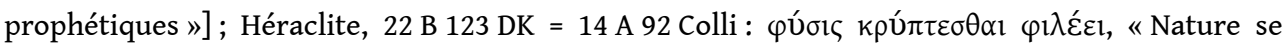

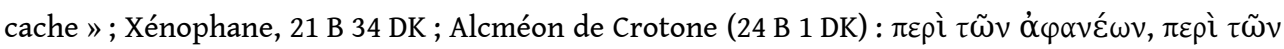

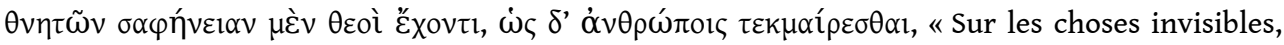
sur les choses mortelles, les dieux détiennent la connaissance immédiate. Mais aux hommes, il est donné de conjecturer. » Il existe donc une fracture entre les dieux et les mortels. Elle n'a rien à voir avec la fracture métaphysique, qui nous est familière, entre un dieu transcendant et ses croyants. Les dieux des Anciens ne sont pas transcendants, cf. e.g. DETIENNE (2007² [1989]), p. 58- 
59. Cette fracture réside dans la différence de condition entre les mortels et les immortels (ceux dont la force vitale, $\alpha i \tilde{\omega} v$, ne s'épuise pas, cf. DETIENNE, ibid., p. 61), mais aussi entre les ignorants et ceux qui ont accès à la connaissance de ce qui est caché, cf. DUHEM (2008 [1908]), p. 32-33. Une manifestation remarquable de cette fracture est la différence de langue entre les dieux et les mortels (cf. CLAY [1972] ; LECLERC [1993]), telle qu'elle apparaît par exemple dans l'épisode de l' Odyssée $(\mathrm{X}, 281-305)$ où Hermès offre à Ulysse le $\mu \tilde{\omega} \lambda v$, mais aussi dans le caractère énigmatique de la parole d'Apollon (cf. colli [1975], p. 49-69).

53. Cf. DETIENNE (1998), p. 52 : Théoclymène prophétise à partir d'un épervier qui emporte dans ses serres une colombe et la dépouille de ses plumes (Od.XV, 525-534); puis il $\mu \alpha v \tau \varepsilon u ́ \varepsilon \tau \alpha l$ à Pénélope qu'Ulysse est là (XVII, 151-161) : il le voit ; puis il prophétise, dans un état possessionnel, aux Prétendants (XX, 351-370).

54. Cf. DETIENNE (2006² [1967]).

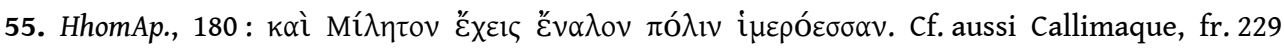

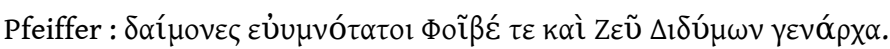

56. Diogène Laërce en donne différentes versions (I, 28-33).

57. 492 F 18 Jacoby (= Diogène Laërce, I, 28). Il est inutile de corriger Leandrios en Meandrios, cf. la note ad loc. de Richard Goulet dans l'édition de Diogène Laërce dirigée par Marie-Odile Goulet-Cazé.

58. Callimaque, Iamb., fr. 191, 56-62 Pfeiffer (= Thalès, 11 A 3a DK = 10 B 7 Colli). Ce témoignage de Callimaque, qui enrichit à la fois notre connaissance de Thalès et de la culture hellénistique, ne figure pas dans l'édition Laks-Most.

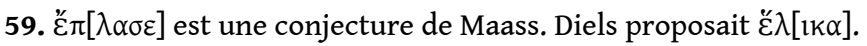

60. Sur cette façon de désigner les Arcadiens, cf. BORGEAUD (1979), p. 19.

61. C'est-à-dire Pythagore. Euphorbe, héros troyen, était l'une des incarnations antérieures que lui attribuait la tradition. Cf. MACRIS (2018), p. 813-814.

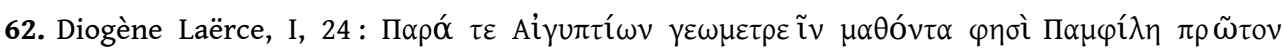

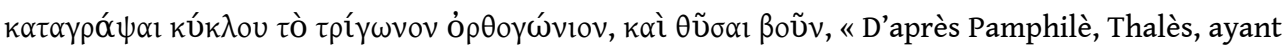
appris auprès des Égyptiens à pratiquer la géométrie, fut le premier à dessiner un triangle rectangle dans un cercle, après quoi il sacrifia un bœuf ». Sur Pamphilè, cf. la notice de R. GOULET dans le Dictionnaire des philosophiques antiques (P 12).

63. HhomAp., 180 (cité supra n. 55).

64. Diogène Laërce, I, 24.

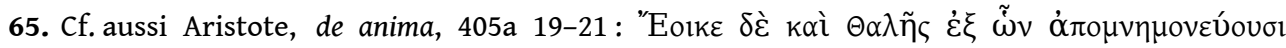

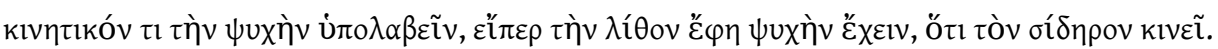

66. DETIENNE (1998), p. 138-150 et p. 170-172.

67. Commenté par DETIENNE (ibid., p. 52-53).

68. Aristote, de anima, 411a 7-8 Ross (= Thalès, 11 A $22 \mathrm{DK}=10 \mathrm{~A} 14$ Colli).

69. Aëtius, Dox. I, 7, 11 (= Thalès, $11 \mathrm{~A} 23 \mathrm{DK}=10 \mathrm{~B} 8 \mathrm{~b}$ Colli).

70. VERNANT (1985 [1965]), p. 380.

71. Plutarque, Sol., 12.

72. Aristote, Pol. I, 4, 1259a 5-18 (= Thalès, 11 A 10 DK = 10 A 10 Colli).

73. Sur la culture des olives à Milet et leur importance économique, cf. les pages extrêmement riches de GREAVES (2002), p. 25-28, qui commente notre passage.

74. NIETZSCHE (1994 [1872]), p. 107.

75. HEIT (2007), p. 38-39.

76. HEIT (2007), p. 39, conclut avec humour : « Wieso eine solche Glückssache, die nur zufällig bestätigt wurde, als Beginn wissenschaftlichen Arbeitens gilt, ist nicht jedem ersichtlich. »Cf. les paroles résignées et ironiques du grand historien de l'astronomie et des mathématiques, Otto E. NEUGEBAUER (1975), p. $604:$ : As a concession to the innumerable discussions concerning the prediction of a solar eclipse in -584 
(May 28) by Thales a few remarks may be made here though I have no doubt that they will remain without effect. [...] It is now evident that even three centuries after Thales no solar eclipse could be predicted to be visible in Asia Minor - in fact not even for Babylon » (je souligne). Sur ce savant exemplaire, notamment face au nazisme, cf. SWERDLOW (1998).

77. FONTENROSE (1988), p. 9 : "The earliest sanctuary of Apollo at Didyma probably consisted of an enclosure about an altar and spring. The first stone structures at Didyma, built in the late 7th century [donc au début de la vie de Thalès], were probably preceded by wooden buildings. Apollo's wooden temple was presumably a small structure, replaced by the first stone naiskos, a little temple or chapel. Then high walls were built to enclose the naiskos. "

78. Crésus l'honora à parts égales avec Delphes.

79. Cf. PARKe (1985), p. 60. D'après Pausanias (VII, 2, 6), l'oracle était là avant la colonisation ionienne. Hérodote le désigne toujours du nom de «Branchides ", ce qui fait penser à PARKE (ibid., p. 59) que les Branchides étaient « in origin one of those communities centred on the sanctuary of a local deity, such as were typical in other parts of Asia Minor before it was hellenized, and which survived in places throughout classical times ", avec aussi des remarques très intéressantes (ibid., p. 59-60), sur le caractère unique du nom Branchos «in Greek mythology » et son sens de " rauque, enroué ». Il s'agirait d'une explication a posteriori d'un nom dérivé d'une langue non hellénique d'Asie mineure.

80. Hérodote, II, 159. Cf. GREAVES (2002), p. 126-127. Il existait des contacts entre Milet et Naucratis (ibid., p. 84), et l'on a retrouvé des objets égyptiens dans le temple d'Aphrodite et dans la « tombe du lion » (ibid., p. 89). Sur le commerce entre Milet et l'Égypte, cf. ibid., p. 101.

81. Sur ces prêtres de Milet, cf. GRAF (1974) ; DETIENNE (1998), p. 209-215 ; GEORGOUdI (2001).

82. Sur ces pierres sacrées, cf. HERDA (2006). Le fait que leur nom ne s'explique par aucun autre mot grec, et soit donc probablement un mot carien, le fait aussi qu'on verse sur elles du vin pur et non du vin mélangé, contrairement aux libations habituelles, peut laisser penser qu'il s'agit d'objets remontant à une période très ancienne, antérieure à la colonisation. Cf. aussi CARBON (2013).

83. Elles sont couronnées et on verse sur elles du vin pur.

84. C'est la première attestation de cette déesse carienne dans un texte grec.

85. On a retrouvé la statue de Charès, mentionné sur l'inscription. C'était un des gouverneurs auxquels Milet déléguait une partie de l'administration de son territoire. Un autre exemple dans Hérodote, avec un archôn d'Assessos, cf. PARKE (1985), n. 8, p. 60.

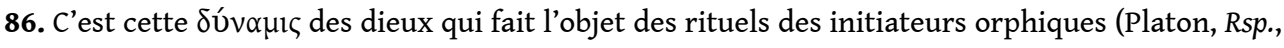

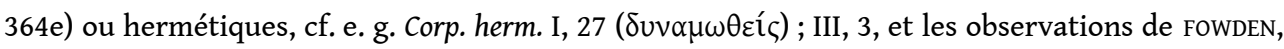
$\left(1993^{2}[1986]\right)$, p. 79

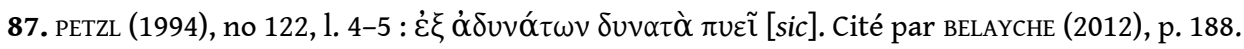

88. Ap. Aristote, Phys. III, 4, 203b, cité par BELAYCHE (2012), p. 187, qui commente ainsi le mot : il « convient bien pour exprimer le monde supérieur conçu prioritairement comme une puissance (dunamis) dont les hommes font l'expérience ici-bas.» Et plus loin (p. 191) : «le Theion est une forme d'expression de la dunamis de tout être supérieur quand elle agit dans le monde. » Mais je n'en conclurais pas que «le terme remonte aux philosophes présocratiques» (p. 193). Comme le

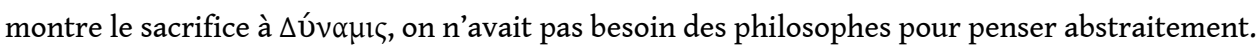

89. ROBERT (1978), p. 21 : «Une fois de plus, un panorama peut livrer la clef d'un site et de son histoire. »Cf. aussi ROBERT (1977), étude sur Kythnos, dans les Cyclades.

90. GÖDECKEN (1986), p. 217 ; « Chronique des fouilles et découvertes archéologiques dans l'Orient hellénique », BCH 48 (1924), p. 468 (village au sud-est de Sparte); Psychiko, dans une banlieue d'Athènes. 
91. Anaxagore, 59 A 7 DK (Clément d'Alexandrie, Strom., I, 63) : il « transporta ( $\mu \varepsilon \tau \eta ́ \gamma \alpha \gamma \varepsilon v)$ d'Ionie à Athènes son enseignement ( $\left.\delta_{1} \alpha \tau \rho \beta \eta ́ v\right) » ;$ Ps-Galien, Hist. phil., 3. En fait, il est peu probable, pour des raisons chronologiques, qu'il ait été disciple d'Anaximène (R. GOULET, DPhA, t. I, p. 184).

92. C'est ainsi que le voit même Nietzsche, par exemple, cf. FP 21 (15) 1872-3 et 23 (8) 1872-3.

93. Cicéron, Div. I, 112.

94. Caelestium litterarum scientia, dit Pline $(2,149)$. Cf. Diogène Laërce, II, 10 ; Anaxagore, 59 A 6 DK ; A 11 ; A 12. On montrait la pierre à l'époque de Pline (II, 149 : celebrant Graeci). Cf. aussi Plutarque, Lys., 12.

95. Philostrate, V.A. I, 2, 6 et Diogène Laërce, II, 10.

96. Toutes prédictions relevées par Pline l'Ancien (II 149 = Anaxagore, 59 A 10 DK), Philostrate (Anaxagore, 59 A $7 \mathrm{DK}$ ), Ammien Marcellin (Anaxagore, 59 A $10 \mathrm{DK}$, avec cette précision intéressante: à partir des livres des Égyptiens et putealem linum contrectans tremores futuros praedixerat terrae).

97. Pline l'Ancien, II, 149 : maioris miraculi diuinitatem Anaxagorae fuisse; Hippolyte, Ref. I, 8, 13 :

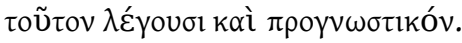

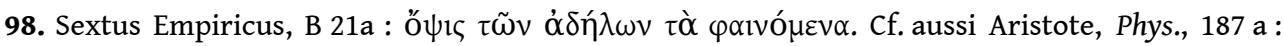
tout est à l'intérieur mais on ne le voit pas.

99. Cf. Xénophane, 21 B 34 DK ; Épiménide, 3 B 11 DK = 8 A 4 Colli ; Héraclite, 22 B 123 DK = 14 A 92 Colli (cité supra n. 52). MARCovich (1965), p. 266 et 277-278, relève dix citations ou adaptations de cette maxime. Cf. enfin le «médecin» pythagoricien Alcméon de Crotone (Diogène Laërce, VIII, 83), cité supra n. 52.

100. Le mot «homéomérie " n'apparaît que dans les témoignages aristotéliciens sur la pensée d'Anaxagore. Certains savants en ont déduit que le terme n'était pas d'Anaxagore. Le débat n'est pas clos. Quoi qu'il en soit, que le mot ait été ou non inventé par Anaxagore est une question secondaire : ce qui compte à mes yeux, comme pour les prédictions, ce n'est pas la question de l'historicité ou de l'authenticité, mais celle de savoir comment on se représentait les actes, actes sociaux, actes de pensée, d'un sage comme Anaxagore.

101. Anaxagore, 59 A 47 DK.

102. Anaxagore, 59 B 12 DK ; Lucrèce, I, 877-878.

103. Anaxagore, 59 A $90 ; 104$; B $10 \mathrm{DK}$.

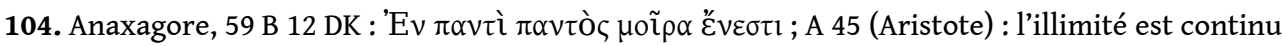

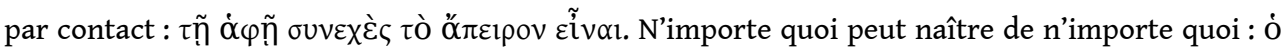

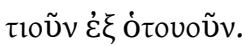

105. Anaxagore, 59 B $21 \mathrm{DK}$

106. Anaxagore, 59 A $97 \mathrm{DK}$ : il oppose vooú $\mu \varepsilon v \alpha$ et phénomènes. La neige est noire, car la neige, c'est de l'eau gelée ; or l'eau est noire ; donc la neige est noire, comme le prouve la couleur noire de la fumée qui s'échappe de l'eau présente dans le bois (scholies A à Iliade XVI, 161 = Anaxagore, 59 A 98 DK).

107. Anaxagore, 59 A $100 \mathrm{DK}$ (Aristote, de anim. I, 2, 404b 1) : Anaxagore est moins explicite sur ce point : la plupart du temps, il dit que le Noûs est la cause de ce qui est beau et correct (donc déçoit Socrate car en même temps cette cause du beau et du correct a l'air de gouverner un cosmos en désordre). Tantôt le Noûs est séparé des choses (B 12), tantôt certaines choses en contiennent une part (B 11), tantôt toutes les choses (par ex. les plantes, A 117).

108. Anaxagore, 59 A $99 \mathrm{DK}$ (= Aristote, de anim. I, 2, 404a 25) : "Anaxagore affirme aussi qu'est âme ce qui meut même si un autre a dit que le Noûs a mis le tout en mouvement » [ce témoignage ne figure pas dans l'édition Laks-Most] ; 59 A 100 DK (=Aristote, de anim. I, 2, 404b 1-7) : « Mais ailleurs il a l'air de dire que le Noûs est l'âme puisque selon lui il appartient à tous les animaux grands et petits, nobles et vils. Or il ne semble pas que le Noûs, pris au sens de faculté 
raisonnable, appartienne de la même façon à tous les animaux, ni même à tous les hommes. » Cf. aussi Diogène Laërce, II, 8 : le Noûs est principe de mouvement.

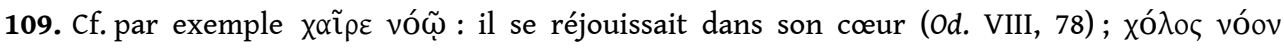

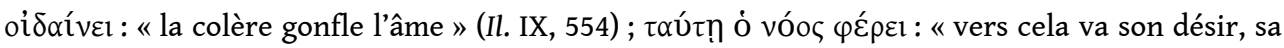

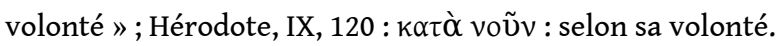

110. Aristote, Protr., fr. 10c Ross (= Jamblique, Protr., 8, p. 54-55 Pistelli) : ò voũ $\zeta$ үà $\rho \dot{\eta} \mu \tilde{\omega} v$ ò $\theta \varepsilon o ́ \varsigma$

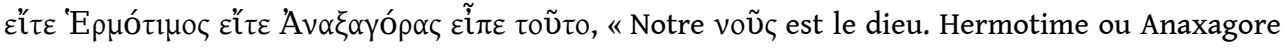
l'a dit »; De anima III, 4, 429a 18 (= Anaxagore, 59 A 100 DK) : le noûs doit être ó $\mu 1 \gamma \tilde{\eta}$ [...] ǐv $\alpha$ kp

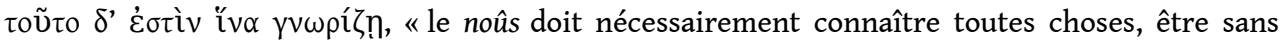
mélange, afin de dominer, c'est-à-dire de connaître »; cf. aussi A 48, fragment reconstitué à partir du de pietate de Philodème de Gadara, 4a, p. 66 Gomperz (= VASSAllo [2019], CPH IV 24) : " [dieu ?] a été, est et sera et gouverne et maîtrise toutes choses ». Ce témoignage de Philodème ne figure pas dans l'édition Laks-Most. Les Anciens avaient reconnu cet enseignement dans

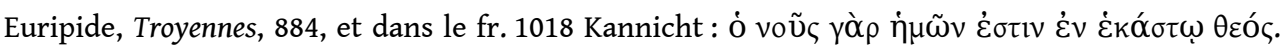
On peut ajouter au témoignage d'Aristote et de Philodème celui de Cicéron, Ac. Pr. II, 118 (= Anaxagore, 59 A 49 DK) : materiam infinitam, sed ex ea particulas similes inter se minutas, eas primum confusas postea in ordinem adductas. Cf. enfin les témoignages tardifs de Simplicius, Commentaire sur

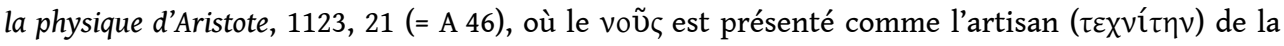
matière, et d'Aetius, Dox. I, 7, 5 (= A 48), où il est question du voũ ৎ du dieu ; un peu plus loin (I, 7,

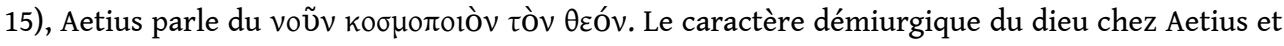
Simplicius ne remonte peut-être pas à Anaxagore, mais il n'y a pas de raison de suspecter l'identification du voũ s̀ une divinité.

111. Aristote, Met. I, 984b 15-20 (= Anaxagore, 59 A 58 DK).

112. Pline l'Ancien, VII, 174 ; Plutarque, Gen. Socr., 22 (Mor., 592c).

113. Cf. Plutarque, Gen. Socr., 22 (Mor., 592c) ; Pline l'Ancien, VII, 174 : reperimus inter exempla Hermotimi Clazomenii animam relicto corpore errare solitam vagamque e longinquo multa adnuntiare, quae nisi a prasente nosci non possent, corpore interim semianimi, donec cremato eo inimici, qui Cantharidae uocabantur, remeanti animae ueluti uaginam ademerint. Cf. aussi Tertullien, de anim., 4344.

114. En témoigne Héraclide du Pont (ap. Diogène Laërce, VIII, 4-5 = fr. 89 Wehrli), qui fait de Pythagore une réincarnation d'Hermotime.

115. Cléarque de Soles, fr. 7 et 8 Wehrli (= Proclus, in Platonis Rem p., II, p. 113, 19 et p. 122, 22 Kroll). Cf. aussi, peut-être, le fr. 11 Ross de l'Eudème d'Aristote, si l'on a raison d'identifier à Hermotime le roi dont parle Al-Kindi (mais cette hypothèse est aujourd'hui rejetée par les spécialistes, cf. DORANDI [2006], p. 19-24). Bien sûr, ce passage a été jugé fictif, cf. e.g. WILAMOWITZMOELLENDORFF (1931-1932), II, n. 1 p. 252-253 ; LEWY (1938). Mais la réhabilitation énergique de Cléarque par Louis ROBERT ([1968], p. 443-448) devrait nous conduire à prendre au sérieux son témoignage. Sur le de somno de Cléarque, cf. DORANDI (2006), qui commente notre passage p. 17.

116. Aristote, Protr., fr. 10c Ross (Jamblique, Protr. VIII, 54-55 Pistelli).

117. Il est significatif que B. CENTRONE, dans la notice du DPhA consacrée à Hermotime, ne cite pas le témoignage de Cléarque.

118. Cf. Phérécyde, 7 B 2 DK = A 2 Colli, le voile brodé offert par Zas à Chtonie.

119. Cf. la cosmogonie révélée par l'extraordinaire fr. 81 Calame, «certainement la plus grande surprise réservée par la publication des P. Oxy. » (p. 439-440).

120. Jamblique, V.P., 14.

121. Sur la «physiologie » possessionnelle d'Épicure, cf. косн-PIETTRE (2005), p. 113-123.

122. Diogène Laërce, II, 12 : procès pour impiété, condamné à amende de 5 talents, banni d'Athènes; Satyros, fr. 14 (FHG III 163) : collaboration avec Mèdes, condamné à mort par contumace. Sur la date du procès, cf. MANSFELD (1979). 


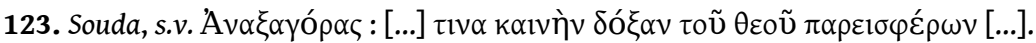

124. Platon, Ap., 26d. Ce prix peu élevé (mais qui pouvait correspondre à une journée de travail) ne veut pas dire que ce sont de mauvaises éditions ou qu'Anaxagore était passé de mode à l'époque de Socrate, mais, plus probablement, que le texte tenait sur un rouleau de papyrus.

125. Cf. les références, toutes tirés de poètes comiques, données par HARVEY (1966), n. 8, p. 634.

126. Eupolis, fr. 327 Kassel-Austin.

127. Cf. Nic., 23.

128. Eschine le socratique ( 59 A $22 \mathrm{DK}=$ Athénée, $\mathrm{V}, 220$ b), dans son Callias, fait la satire des sophistes Prodicos et Anaxagore. Il est significatif que les éditeurs veuillent parfois remplacer Anaxagore par Protagoras. Dans l'édition Laks-Most, le témoignage ne figure pas dans le chapitre consacré à Anaxagore, mais seulement dans celui consacré à Prodicos. Cf. aussi Diodore de Sicile, XII, 39 (= Anaxagore, 59 A 17 DK) ; Harpocration, Lexique, s.v. (= Anaxagore, 59 A 2 DK). Ce dernier témoignage ne figure pas dans l'édition Laks-Most.

129. Cf. Pér., 16.

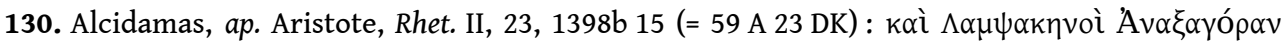

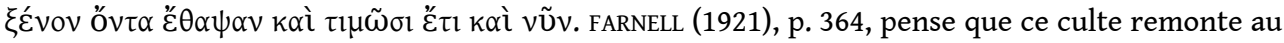
$\mathrm{V}^{\mathrm{e}}$ siècle, à juste titre puisque Alcidamas à la fin $\mathrm{du} \mathrm{v}^{\mathrm{e}}$ ou au début $\mathrm{du} \mathrm{IV}^{\mathrm{e}}$, dit : «aujourd'hui

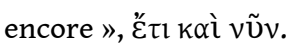

131. Élien, VH, 8, 19 (= 59 A 24 DK). Le texte est incertain. On peut aussi comprendre que l'autel était consacré soit au Noûs, soit à la Vérité. Cf. CURD (2007), n. 10, p. 88.

132. Cette pratique était beaucoup plus courante qu'on ne l'a cru, comme l'a bien montré le livre de CURRIE (2005). Outre Archiloque (cf. note précédente), cf. aussi Hésiode à Orchomènes (Paus., IX, 38, 3 : épidémie stoppée par acquisition de ses os) ; Aristéas (Apollonius, Hist. mirab., 2, p. 44 Keller = Hyperborei, B 1 Colli : un temple et des sacrifices héroïques en Sicile) ; Pindare (Pausanias, IX, 23, 3 : part de sacrifices à Delphes) ; « only a $\mu v \tilde{\eta} \mu \alpha$ » à Thèbes (Vit. Pind., p. 92 Westermann). Sur l'héroïsation du theios aner, cf. CURRIE (2005), fin de la $2^{\mathrm{e}}$ partie. Les habitants de Clazomènes auraient de même rendu un culte à Hermotime, si l'on en croit Tertullien, de anim., 2 et 44.

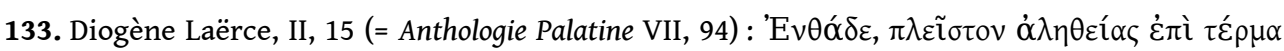

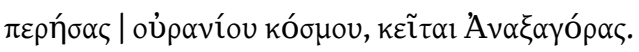

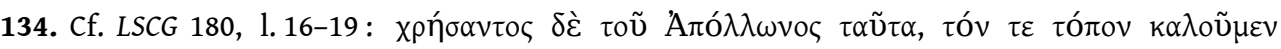

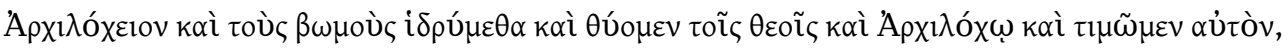

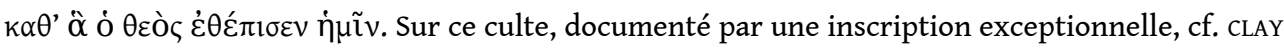
(2004).

135. Diogène Laërce, II, 14-15.

136. Diogène Laërce, IX, 3

137. Ibid., 7.

138. La traduction par «tu ne saurais mieux dire ", dans l'édition Dumont de «La Pléiade » est donc un contresens. Sur l'euphemia, cf. GÖDDE (2011).

139. Cf. aussi Eth. Eud. I, 5, 1216a 11 : un homme ne savait pas répondre au problème; en vue de

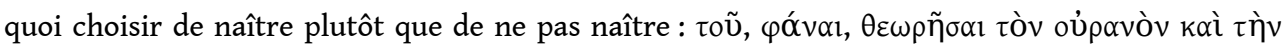

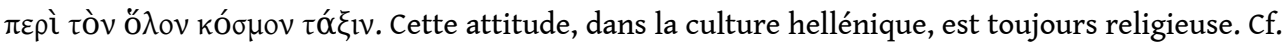

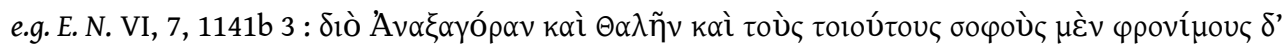

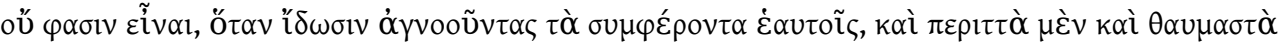

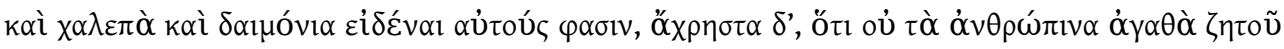
olv.

140. Sur ce point, cf. les nombreux témoignages rassemblés par Pierre BOYANCÉ dans son Culte des Muses (1937), notamment p. 168-176, 215-225, 264.

141. Comme l'épisode du bélier unicorne dans Plutarque, Per., 6, qui met aux prises Anaxagore et le devin Lampon. 


\section{ABSTRACTS}

Reading the extant testimonia on the so-called "Ionian School" from a historical-anthropological perspective, this paper tries to challenge the idea, still domineering in the field of Classical Studies or Ancient Philosophy, that "philosophy" was born in Ionia in the $6^{\text {th }}$ century BC, through a transition "from Myth to Reason", from a "religious" to a "scientific" form of thought. The testimonia suggest instead that the "Ionians" wisdom and knowledge (sophia has the two meanings) cannot be acquired nor activated without the gods. The two figures examined are the ones about whom we have enough documentation to draw conclusions : Thales, the "founder" of the school, and Anaxagoras, who is said to have passed its spirit to Athens.

À partir d'une anthropologie historique des témoignages sur la sophia de Thalès et Anaxagore, cet article tente de discuter l'idée, encore dominante dans l'historiographie courante de la philosophie, d'une "naissance de la philosophie» en Ionie au $\mathrm{vI}^{\mathrm{e}}$ siècle, coïncidant avec un passage du «mythe à la Raison», du "religieux» au «scientifique». Le savoir-sagesse des « Ioniens ", $y$ compris dans ses aspects que nous définirions comme " rationnels », ne peut se pratiquer que dans la dépendance des dieux. Les deux figures étudiées sont celles pour lesquelles les témoignages sont suffisamment nombreux: il s'agit de Thalès, le fondateur de l'école, et d'Anaxagore, qui en aurait transmis l'esprit à Athènes.

\section{AUTHOR}

\section{PIERRE VESPERINI}

Instituto de Filosofia, Université de Porto

pvesperini@gmail.com 